\title{
An Overall Uniformity Optimization Method of the Spherical Icosahedral Grid Based on the Optimal Transformation Theory
}

\author{
Fuli Luo, Xuesheng Zhao*D, Wenbin Sun, Yalu Li and Yuanzheng Duan \\ School of Geoscience and Surveying Engineering, China University of Mining and Technology (Beijing), \\ D11 Xueyuan Road, Beijing 100083, China; lf1@student.cumtb.edu.cn (F.L.); swb@cumtb.edu.cn (W.S.); \\ bqt1700205021@student.cumtb.edu.cn (Y.L.); bqt1900205059@student.cumtb.edu.cn (Y.D.) \\ * Correspondence: zxs@cumtb.edu.cn
}

check for updates

Citation: Luo, F.; Zhao, X.; Sun, W.; Li, Y.; Duan, Y. An Overall Uniformity Optimization Method of the Spherical Icosahedral Grid Based on the Optimal Transformation Theory. Atmosphere 2021, 12, 1516. https:// doi.org/10.3390/atmos12111516

Academic Editor: Tomasz Gierczak

Received: 22 September 2021

Accepted: 14 November 2021

Published: 17 November 2021

Publisher's Note: MDPI stays neutral with regard to jurisdictional claims in published maps and institutional affiliations.

Copyright: (c) 2021 by the authors. Licensee MDPI, Basel, Switzerland. This article is an open access article distributed under the terms and conditions of the Creative Commons Attribution (CC BY) license (https:/ / creativecommons.org/licenses/by/ $4.0 /)$.
Abstract: The improvement of overall uniformity and smoothness of spherical icosahedral grids, the basic framework of atmospheric models, is a key to reducing simulation errors. However, most of the existing grid optimization methods have optimized grid from different aspects and not improved overall uniformity and smoothness of grid at the same time, directly affecting the accuracy and stability of numerical simulation. Although a well-defined grid with more than 12 points cannot be constructed on a sphere, the area uniformity and the interval uniformity of the spherical grid can be traded off to enhance extremely the overall grid uniformity and smoothness. To solve this problem, an overall uniformity and smoothness optimization method of the spherical icosahedral grid is proposed based on the optimal transformation theory. The spherical cell decomposition method has been introduced to iteratively update the grid to minimize the spherical transportation cost, achieving an overall optimization of the spherical icosahedral grid. Experiments on the four optimized grids (the spring dynamics optimized grid, the Heikes and Randall optimized grid, the spherical centroidal Voronoi tessellations optimized grid and XU optimized grid) demonstrate that the grid area uniformity of our method has been raised by $22.60 \%$ of SPRG grid, $-1.30 \%$ of HR grid, $38.30 \%$ of SCVT grid and $38.20 \%$ of XU grid, and the grid interval uniformity has been improved by $2.50 \%$ of SPRG grid, $2.80 \%$ of HR grid, $11.10 \%$ of SCVT grid and $11.00 \%$ of XU grid. Although the grid uniformity of the proposed method is similar with the HR grid, the smoothness of grid deformation has been enhanced by $79.32 \%$ of grid area and $24.07 \%$ of grid length. To some extent, the proposed method may be viewed as a novel optimization approach of the spherical icosahedral grid which can improve grid overall uniformity and smoothness of grid deformation.

Keywords: optimal transformation theory; uniform grid; optimized spherical icosahedral grid; area uniformity; interval uniformity

\section{Introduction}

Spherical icosahedral grid is often used as the basic framework of atmospheric models, such as the ICosahedral Nonhydrostatic model (ICON) [1-3], Models for Predication Across Scales (MPAS) [4], the Non-hydrostatic ICosahedral Atmospheric Model (NICAM) [5], DYNAMICO [6], Ocean-Land-Atmosphere Model (OLAM) [7,8], etc. These models are wieldy used for polar lows simulation [9], monsoon simulation [10], ocean tides dissipation [11], etc. Recently, the Spectral Radiation Transport Model for Aerosol Species coupled with NICAM (NICAM-SPRINTARS) [12] has been developed for studying and simulating atmosphere-aerosols interactions and their effects on atmospheric pollution and climate change. Related studies include simulating the annual aerosol characteristics over China [13], capturing the horizontal distribution of aerosol optical thickness fields [14], studying the resolution dependency of the transport process of black carbon from Siberia to Japan [15], and assimilating global aerosol vertical observations [16], among others.

The accuracy of numerical simulations is affected by the quality of the spherical icosahedral grid [17-19]. Low-quality grids may cause large numerical approximation errors. 
Some noises are leaded by slight irregularities of the grid, which the long-term simulation will become unstable [20]. This is because nonuniform grids may bring grid imprinting in the numerical solutions and speed up their spread in meteorological models [21,22]. In this way, the nonuniformity of the control volume becomes the main source of numerical simulation error [23]. For example, the non-orthogonality of the grid edge and its dual edge, and the non-coincidence of the midpoints of the two edges increases the truncation error of the spherical differential operators [22,23]. Grid misalignment also leads to errors in some low-order algorithms of the spherical surface $[19,24]$, resulting in discretization of differential operators to only meet first-order convergence [17]. The grid with good quality can reduce the truncation error of these operators on the sphere [25].

At present, some grid optimization methods have been proposed to improve grid quality and to reduce the simulation errors [26]. The spring dynamics method (i.e., the SPRing dynamics Grid optimization (SPRG) method) $[27,28]$ is utilized to improve grid interval uniformity and to reduce geometric variations [19]. Iga [29] found that the interval distributions of grids near the icosahedron vertices, optimized by the SPRG method with a zero natural spring length (SPR0), are inversely proportional to the map factor of the Lambert conformal conic projection. An analytical transformation was proposed to stretch the grids near the icosahedral vertices to decrease grid aggregations [30]. Heikes and Randall [31,32] (HR) have minimized the distances between the midpoints of hexagonal/pentagonal grids edges and their dual (triangles) edges to optimize grid. Then, they have re-optimized the grid (named tweaked grid [33]) based on previous result to avoid the hemispheric twisting, where the grid quality and the convergence of PDE operators have been further improved. Spherical Centroidal Voronoi Tessellations (SCVT) [34-36] has been put forward, in which grids' centers were iteratively moved by Llyod's method [37] to their mass centroids to minimize the distances between these two points. $\mathrm{Xu}$ [25] has updated iteratively grids based on the Laplace-Beltrami solution, and the optimization grid and SCVT grid are very close to each other.

Comparations have been done with the main optimization grids, such as HR grid, SCVT grid, SPRG grid, etc. for grid quality $[23,33]$. Some indicators, for instance the ratios between minimum and maximum grid area, the ratios between minimum and maximum grid intervals, the grid area ranges, grid interval ranges, area relative deviations, length relative deviations, etc. are used to evaluate grid quality. The grid interval ratio of SPRG grid is bigger than HR grid, that means SPRG grid's interval uniformity is better than that of HR grid. However, its grid area ratio and grid interval ratio are all less than the nonoptimized grid (NOPT grid). Its grid quality is limited by the spring constant. When the spring constant is large, some grids near the icosahedral vertices may collapse at high resolutions. When the constant is small, grids do not collapse, but some grids near the icosahedral vertices may aggregate, such that grid intervals are shortened and the amount of calculation is increased [29]. The grid intervals by Iga [29] only be increased along the stretch paths, decreasing the regularity of the grids near the icosahedral vertices. Although area uniformity of HR gird has been enhanced to the most extent, its grid interval ratio is slightly smaller than SPRG grid and NOPT grid. Among these grids, the quality of SCVT grid is the worst, its two ratios are divergent with the increasing grid resolution, and discretization accuracy of basic operator is also lowest. There is none of them being clearly better than the others [19].

Tomita [27] has implied that simulation error appears in regions with high gradient of grid area and interval deviations, and the smoothness is important for high-accuracy and stable simulation [29]. The node interpolation can follow a second-order convergence on a uniform spherical grid, but if there is any deformation, it has only a first-order convergence [38]. The grid uniformity is also important to determine the maximum time step for numerical integration [20] and to maintain the consistency of physical parameterization in atmospheric simulations [23]. Meanwhile, improvement of the uniformity is helpful for wavelet transform to improve the compression technique for weather and climate data $[39,40]$. The grid uniformity has been quantified from grid area and grid interval devi- 
ations in some reviews $[20,23,33]$. The grid area uniformity and interval uniformity are not independent of each other. As the spherical surface is non-Euclidean, a well-defined grid with more than 12 points, meting the two attributes simultaneously, cannot be constructed on a sphere [20]. However, the area uniformity and the interval uniformity of the spherical grid can be trade off to enhance extremely the overall grid uniformity and smoothness of the spherical distribution of the grid area and interval deviation.

The present study is devoted to the investigation of a novel grid overall uniformity and smoothness optimization approach rooted in the optimal transportation theory. The spherical cell decomposition method was introduced to iteratively update the grid to minimize the spherical transportation cost, achieving an optimization grid. We discuss the details of the proposed method and its effectiveness from the grid geometry quality and numerical accuracy. Unlike existing optimization approaches, the proposed method is optimal in that it does not only improve the grid overall uniformity but also reduce the grid deformation, enhancing the smoothness of grid deformation. Both the smoothness of deformation and uniformity of grid interval have been greatly improved. The smoothness of grid area deformation has also been heightened even the grid area uniformity is comparable to those obtained by the Heikes and Randall grid.

The rest of this paper is organized as follows: the optimal transportation theory, grid uniformity optimization method and the core algorithm are introduced in the Theory and Methods section. The Results and Discussions section presents the comparative results and discussions from the grid quality and numerical accuracy. The conclusions are shown in the last section.

\section{Theory and Methods}

Optimal transportation theory is used for the measure-preserving mapping between two probability measure spaces, such that the target probability measure is infinitely close to the source (or true) probability measure. In all measure-preserving mappings, a mapping minimizing the transportation cost is an optimal transportation mapping [41]. Gu [41] proposed a discrete spherical optimal transportation mapping based on a purely geometric method and defined the measures as areas to achieve an area-preserving mapping from the topological sphere to the unit sphere [42,43]. The mapping is global and has been applied in biomedicine [44,45], face recognition [46], generative adversarial networks [47], and other fields.

Given a continuous space $\mathbf{R}^{\mathrm{n}}$, two subspaces with measures, $(\boldsymbol{X}, \psi) \in \mathbf{R}^{\mathrm{n}}$ and $(\boldsymbol{Y}, \varphi) \in \mathbf{R}^{\mathrm{n}}$, having the same total measures, that is $\int_{X} \psi d x=\int_{Y} \varphi d y$, there is a measure-preserving mapping $T: X \rightarrow \boldsymbol{Y}$ to make $\varphi(B)=\psi\left(\mathrm{T}^{-1}(B)\right)$, where $B \subset \boldsymbol{Y}, \mathrm{T}^{-1}(\mathrm{~B}) \subset \boldsymbol{X}$. Then the transportation cost can be formalized as in Equation (1):

$$
C(T)=\int_{X} c(x, T(x)) d \psi(x)
$$

where $C: X \times Y \rightarrow R$ is a cost function of $T ; T_{o p t}=\operatorname{argmin}\{C(T)\}$ is the optimal transportation mapping.

Spherical optimal transportation mapping realizes the area-preserving mapping from the topological sphere to the unit sphere. There is a probability measure $\psi$ in the continuous space defined on the unit sphere and a Dirac measure $\varphi=\left\{\varphi_{1}, \varphi_{2}, \ldots, \varphi_{n v}\right\}$ corresponding to the point set $\left\{p_{i}\right\} \subset \mathrm{S}^{2}$, which cannot be covered by any hemisphere, such that $\psi\left(S^{2}\right)=\sum_{i=1}^{n v} \varphi_{i}$. A set of cells, $\boldsymbol{W}=\left\{w_{i}\right\}$, decomposing the unit sphere, that is $S^{2}=\cup_{i=1}^{n v} w_{i}$, can be found to make $\varphi\left(w_{i}\right)=\psi_{i}$. Then a mapping $T: w_{i} \rightarrow p_{i}$ is the optimal transportation mapping minimizing the transportation cost. The existence and uniqueness of the complete solutions in the continuous space have been proved by $\mathrm{Gu}$ (2013). Surfaces can be discretized by triangular meshes. The discrete spherical optimal transportation mapping is an approximate preserved area mapping from a topological spherical triangular mesh to a spherical triangular mesh. A topological spherical triangular mesh is $\boldsymbol{M}\{\boldsymbol{V}, \boldsymbol{E}, \boldsymbol{F}\}$, with point set $\boldsymbol{V}=\left\{v_{1}, v_{2}, \ldots, v_{n v}\right\}$, edge set $\boldsymbol{E}=\left\{e_{1}, e_{2}, \ldots, e_{n e}\right\}$ 
and face set $\boldsymbol{F}=\left\{f_{1}, f_{2}, \ldots, f_{n f}\right\} . \forall v_{i} \in \boldsymbol{V}$, there is a set of first-order adjacent vertices $v_{a d j}=\left\{v_{i_{1}}, v_{i_{2}}, \ldots, v_{i_{N s}}\right\}, N s$ is the number of first-order adjacent vertices. $\forall \psi_{i} \in \boldsymbol{\psi}$, it can be defined as one-third of total area of triangles formed by $v_{i}$ and $v_{a d j}$, its construction is illustrated in Figure 1 and its formulization is defined in Equation (2),

$$
\psi_{i}=\frac{1}{3} \sum_{j=1}^{N s} S_{\Delta v_{i} v_{j} v_{j+1}}
$$

where $S$ is the spherical area of $\Delta v_{i} v_{j} v_{j+1}$.

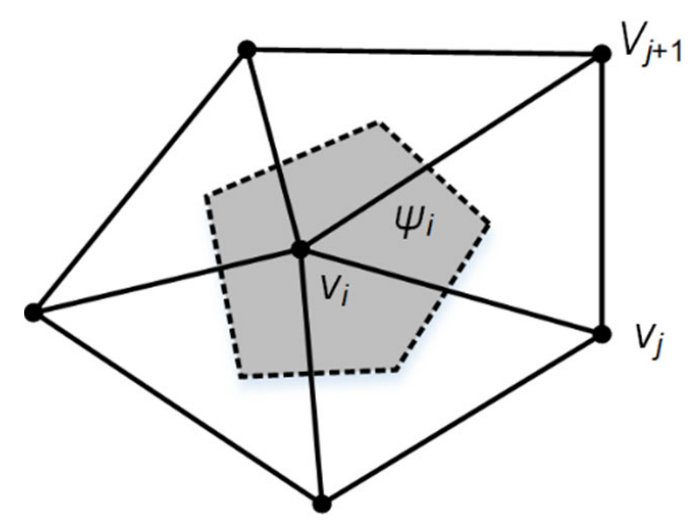

Figure 1. Area measure $\psi_{i}$ of $v_{i}$.

There is an optimal transportation mapping $T:(\boldsymbol{M}, \psi) \rightarrow(\boldsymbol{N}, \boldsymbol{\varphi})$, such that $\varphi_{i}=\psi_{i}$ and $C(T)$ in Equation (3) is minimal, in which $N\left\{\boldsymbol{V}^{*}, \boldsymbol{E}, \boldsymbol{F}\right\}$ is the image of $\boldsymbol{M}, \boldsymbol{\varphi}$ is a measure set of $N$.

$$
C(T)=\frac{1}{2} \sum_{i=1}^{n v}\left(\varphi_{i}-\psi_{i}\right)^{2}
$$

Based on the point set $V$, a cell decomposition set $W=\left\{w_{i}\right\}$ can be constructed, where $w_{i}$ 's area is $\varphi_{i}$. When $\varphi_{i}$ equals $\psi_{i}$, the transportation cost is minimal equal to zero. Updating the center of cell $w_{i}$ as $v_{i}^{*}$ obtains the image of the approximate preserved area mapping.

Cell decomposition on a sphere is a spherical power diagram generation. There is a point set $V=\left\{v_{1}, v_{2}, \ldots, v_{n v}\right\} \in \mathrm{S}^{2}$ and its weight set $r=\left\{r_{1}, r_{2}, \ldots, r_{n v}\right\} \in \mathrm{R}, c_{i}\left(v_{i}, r_{i}\right)$ is a circle on a sphere with center $v_{i}$ and radius $r_{i}$. Spherical power distance between any point $p \notin V$ and $c_{i}$ is defined in Equation (4):

$$
\operatorname{pow}\left(p, v_{i}\right)=\frac{\cos d\left(p, v_{i}\right)}{\cos r_{i}}
$$

where $d(\cdot)$ means the spherical great circle, pow $\left(p, v_{i}\right)$ is a geodesic distance between $p$ and tangent point, intersection of a line through $p$ tangent to circle and the circle, as shown in Figure 2 .

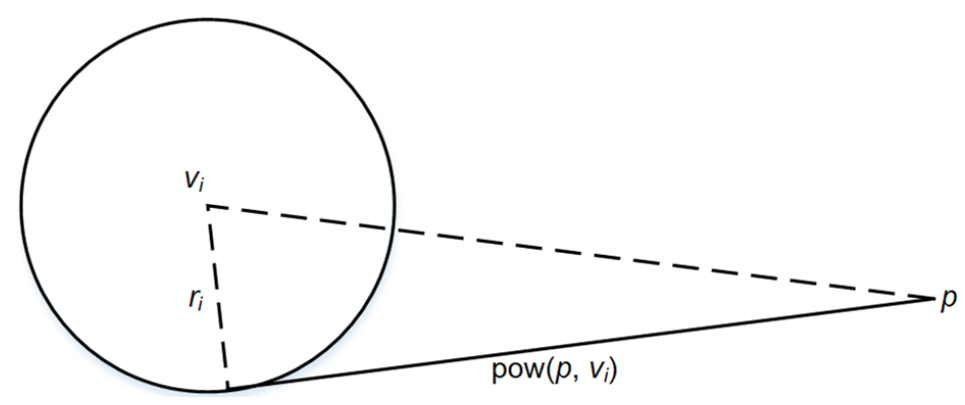

Figure 2. Spherical power distance. 
The spherical power diagram of $\left\{\left(p_{i}, r_{i}\right)\right\}$ is a cell decomposition of sphere, that is $S^{2}=\cup_{i=1}^{n v} w_{i}$, where $w_{i}=\left\{v_{i} \in \boldsymbol{V} \mid \operatorname{pow}\left(p, v_{i}\right)<\operatorname{pow}\left(p, v_{j}\right), \forall v_{j} \in \boldsymbol{V}-\left\{v_{i}\right\}\right\}$. A spherical power diagram of a random point set is shown in Figure 3.

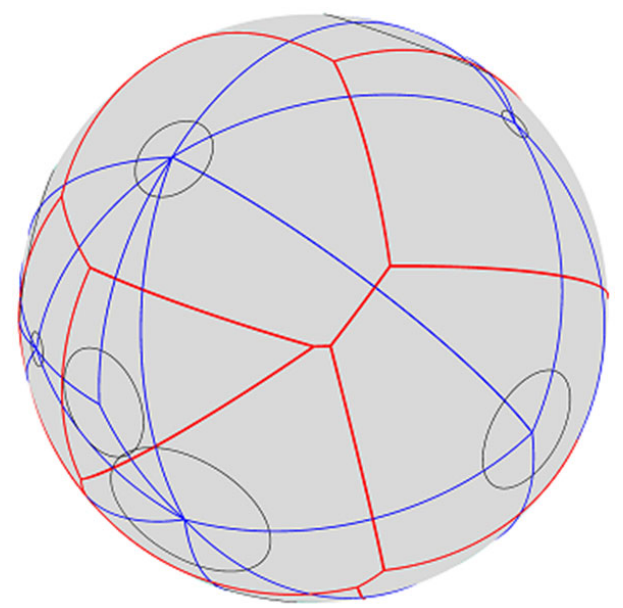

Figure 3. Spherical power diagram.

Where the green circle is spherical circle of each point with center $v_{i}$ and radius $r_{i}$, the red spherical polygon is spherical power cell of each point, and the blue spherical triangle is cell's dual triangle.

It has been proved that the optimal transportation mapping can be achieved by adjusting the weight of the spherical power diagram. When the area of each power cell is equal the predefined weight, defined by the area measure, the optimal transportation mapping can be obtained. According to the spherical power diagram, the transportation cost can be defined as follows:

$$
C(\boldsymbol{h})=\frac{1}{2} \sum_{i=1}^{n v}\left(\varphi\left(\omega\left(h_{i}\right)\right)-\psi_{i}\right)^{2}
$$

where $h$ is a function of radius, $h=-\ln (\cos (r)), \varphi$ is the power cell area.

The power cell area is an analytic function of radius [42]. Let $q$ is a center point of line between $v_{i}$ and $v_{j}$, there is pow $\left(q, v_{i}\right)=$ pow $\left(q, v_{j}\right)$ (Figure 4$)$, then:

$$
\cos d\left(q, v_{i}\right) e^{h_{i}}=\cos d\left(q, v_{j}\right) e^{h_{j}}
$$

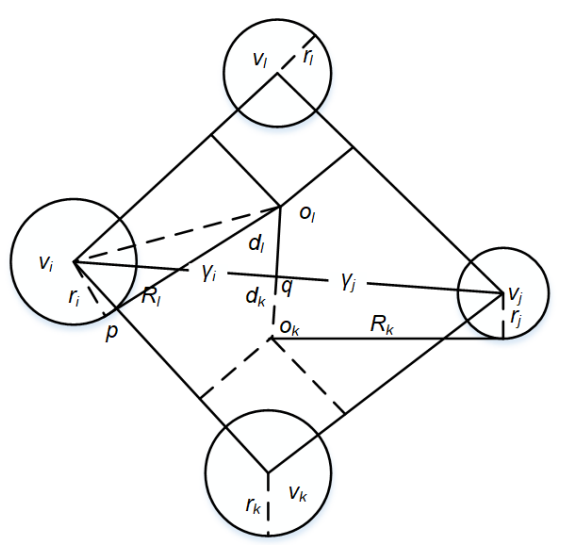

Figure 4. Power cell and its dual triangle. 
Where $R_{l}$ and $R_{k}$ are triangle power radius of $\Delta v_{i} v_{j} v_{k}$ and $\Delta v_{i} v_{j} v_{l} . d_{l}$ and $d_{k}$ are vertical distances from the triangle center $o_{l}$ and $o_{k}$ to edge $\left[v_{i}, v_{j}\right]$, respectively. $\gamma_{i}$ and $\gamma_{j}$ are the distances between $v_{i}$ and $q, v_{j}$ and $q$, respectively.

Let $\gamma_{i}=d\left(q, v_{i}\right), \gamma_{j}=d\left(q, v_{j}\right)$, and $\gamma_{i}+\gamma_{j}=\gamma_{i j}$, there is:

$$
\begin{gathered}
\cos \left(\gamma_{i j}-\gamma_{j}\right) e^{h_{i}}=\cos \gamma_{j} e^{h_{j}} \\
\tan \gamma_{j}=\frac{1}{\sin \gamma_{i j}}\left(e^{h_{j}-h_{i}}-\cos \gamma_{j}\right)
\end{gathered}
$$

According to the partial derivative of $\tan \gamma_{j}$ with respect to $h_{i}$, there is:

$$
\begin{aligned}
\frac{d \gamma_{j}}{d h_{i}} & =\cos ^{2} \gamma_{j} \frac{\partial \tan \gamma_{j}}{\partial h_{i}}=-\cos ^{2} \gamma_{j} \frac{1}{\sin \gamma_{i j}} e^{h_{j}-h_{i}} \\
& =-\frac{1}{\sin \gamma_{i j}} e^{-h_{j}-h_{i}}\left(\cos ^{2} \gamma_{j} e^{h_{j}}\right)^{2}=-\frac{1}{\sin \gamma_{i j}} \cos r_{i} \cos \gamma_{j}\left(\frac{\cos R_{l}}{\cos d_{l}}\right)^{2}
\end{aligned}
$$

According to area of infinitesimal spherical quadrilateral, $\varphi_{i}^{j l}=\operatorname{area}\left(w_{i} \cap \Delta v_{i} v_{j} v_{l}\right)$, $\varphi_{i}^{j k}=\operatorname{area}\left(w_{i} \cap \Delta v_{i} v_{j} v_{k}\right), \varphi$ is the power cell area. There is:

$$
\begin{gathered}
\frac{\partial \varphi_{j k}}{\partial h_{i}}=-\frac{\cos ^{2} R_{l} \sin d_{l}}{\sin \gamma_{i j} \cos ^{2} d_{l}} \cos r_{i} \cos r_{j} \\
\frac{\partial \varphi_{i}^{i k}}{\partial h_{j}}=-\frac{\cos ^{2} R_{k} \sin d_{k}}{\sin \gamma_{i j} \cos ^{2} d_{k}} \cos r_{i} \cos r_{j} \\
\frac{\partial \varphi_{i}}{\partial h_{j}}=\frac{\partial \varphi_{j}}{\partial h_{i}}=-\frac{\cos r_{i} \cos r_{j}}{\sin \gamma_{i j}}\left(\frac{\cos ^{2} R_{l} \sin d_{l}}{\cos ^{2} d_{l}}+\frac{\cos ^{2} R_{k} \sin d_{k}}{\cos ^{2} d_{k}}\right)
\end{gathered}
$$

The convexity of the transportation cost has been proved by Gu (2013) and it can be minimized by means of Newton's method. The gradient of cost can be defined as $\nabla C=\left(\varphi\left(w_{1}\right)-\psi_{1}, \varphi\left(w_{2}\right)-\psi_{2}, \ldots, \varphi\left(w_{n v}\right)-\psi_{n v}\right)$, and the Hessian matrix can be expressed as follows:

$$
H=\left[\begin{array}{cccc}
-\sum_{i \neq 1} \frac{\partial \varphi_{i}}{\partial h_{1}} & \frac{\partial \varphi_{2}}{\partial h_{1}} & \cdots & \frac{\partial \varphi_{n v}}{\partial h_{1}} \\
\frac{\partial \varphi_{1}}{\partial h_{2}} & -\sum_{i \neq 2} \frac{\partial \varphi_{i}}{\partial h_{2}} & \cdots & \frac{\partial \varphi_{n v}}{\partial h_{2}} \\
\vdots & \vdots & \ddots & \vdots \\
\frac{\partial \varphi_{1}}{\partial h_{n v}} & \frac{\partial \varphi_{2}}{\partial h_{n v}} & \cdots & -\sum_{i \neq n v} \frac{\partial \varphi_{i}}{\partial h_{n v}}
\end{array}\right]
$$

For the spherical icosahedral grid, the measure-preserving mapping becomes a selfmapping. The measure is replaced by a virtual measure $\psi_{\text {virtual }}$, where $\psi_{\text {i_virtual }}=4 \pi R^{2} / n v$ for any spherical icosahedral grid point; $R$ is the earth radius. There is a mapping $T$ : $\left(\right.$ Ico-Grid, $\left.\psi_{\text {virtual }}\right) \rightarrow($ AREA-Ico-Grid, $\varphi)$, where Ico-Grid is composed of a grid point set $V=\left\{v_{i}\right\}$, a grid edge set $E$, a grid set $\boldsymbol{F}$ and a grid point set of AREA-Ico-Grid, $V^{*}=\left\{v_{i}^{*}\right\}$ is the image of $V$ under the mapping $T$, its grid edge set and grid set are the same as Ico-Grid's. When the areas of the grids by cell decomposition are equal to each other, that is $\varphi\left(v^{*}\right)=\psi_{\text {virtual }}\left(\mathrm{T}^{-1}\left(v^{*}\right)\right), v^{*} \subset$ AREA-Ico-Grid, $T^{-1}\left(v^{*}\right) \subset$ Ico-Grid, $T$ is the approximate area-preserved mapping. The Algorithm 1 of area quasi-uniformity optimization for the spherical icosahedral grid is described as follows. 


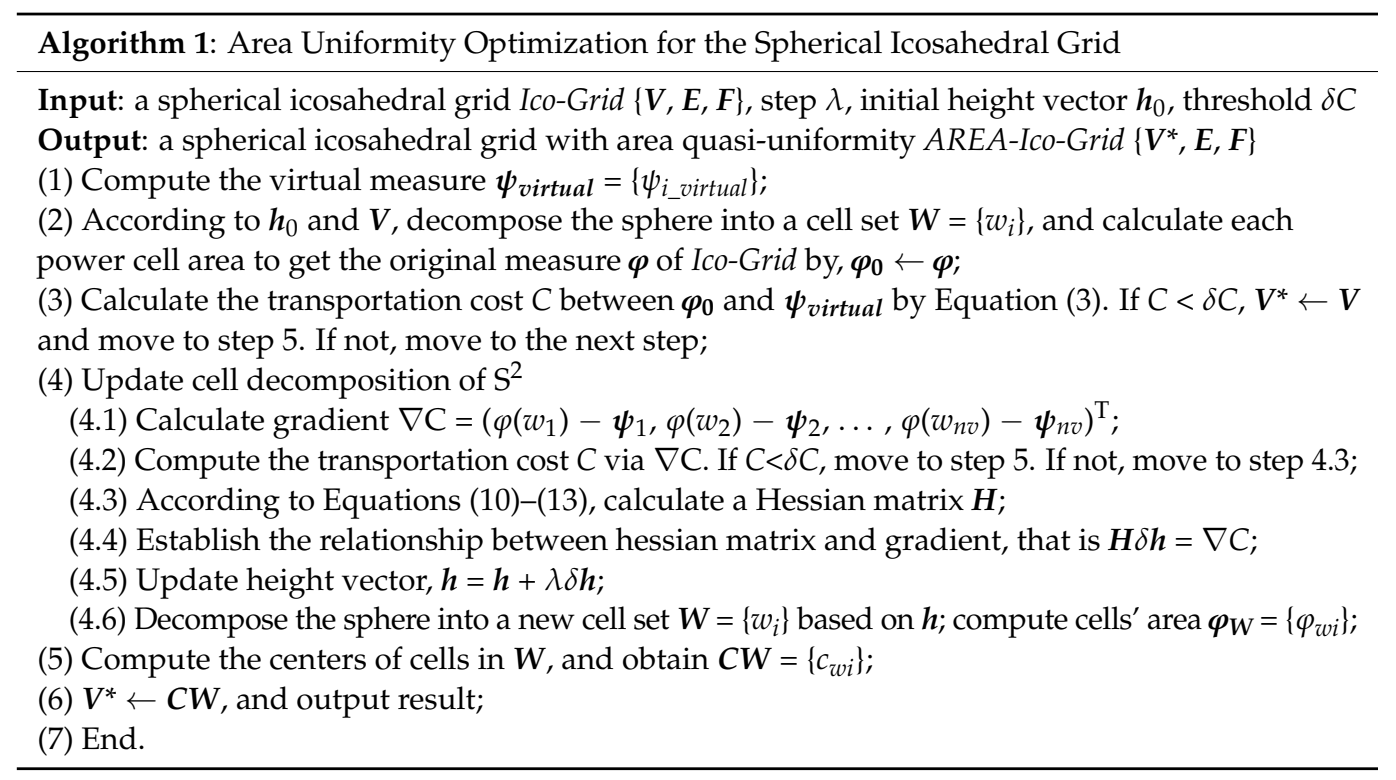

\section{Results and Discussions}

To verify the effectiveness of this algorithm, tests were performed to compare the grid quality and numerical accuracy among the proposed algorithm optimized grid (OURS grid), the non-optimized grid (NOPT grid), the spring dynamic method optimized grid (SPRG grid), tweaked grid optimized by Heikes and Randall grid (HR grid) Spherical Centroidal Voronoi Tessellations grid (SCVT grid) and grid optimized by Xu (XU grid). Here, the construction of the NOPT grid was through recursive division, and the parameter $\beta$ in the SPRG grid was set to 1.1 according to [33]. All experiments were implemented in $\mathrm{C}++$ and executed on a PC with Intel Core i5-6400 CPU@2.70 GHz, 8 GB RAM.

\subsection{The Grid Quality Evaluation}

\subsubsection{The Grid Area Uniformity}

The relative area deviation $D_{\text {area }}$ was used to measure the grid area uniformity, which can be calculated with Equation (14):

$$
D_{\text {area }}=\frac{A-A_{\mathrm{avg}}}{A_{\mathrm{avg}}}
$$

where $A$ is the area of a grid; $A_{\text {avg }}$ is the average area of the spherical icosahedral grid; earth radius was set to $6,371.007 \mathrm{~km}$.

Maximum grid area $A_{\max }$ and minimum grid area $A_{\min }$ and average area $A_{\text {avg }}$ of NOPT grid and OURS grid at certain resolutions are listed in Table 1.

Table 1. $A_{\min }, A_{\max }$ of spherical icosahedral grids (unit: $\mathrm{km}^{2}$ ).

\begin{tabular}{ccccccc}
\hline \multirow{2}{*}{ Level } & \multirow{n}{*}{$\boldsymbol{*} \boldsymbol{A}_{\text {avg }}$} & \multicolumn{2}{c}{ NOPT } & \multicolumn{2}{c}{ OURS } \\
\cline { 3 - 7 } & & & $A_{\min }$ & $A_{\max }$ & $A_{\min }$ & $A_{\max }$ \\
\hline 0 & 12 & $42,505,466.06$ & $42,505,466.06$ & $42,505,466.06$ & $42,505,466.06$ & $42,505,466.06$ \\
1 & 42 & $12,144,418.88$ & $11,115,261.91$ & $12,556,071.10$ & $11,115,254.48$ & $12,556,081.85$ \\
2 & 162 & $3,148,553.04$ & $2,812,532.18$ & $3,339,347.67$ & $2,885,170.41$ & $3,216,806.64$ \\
3 & 642 & $794,494.69$ & $705,296.93$ & $923,852.78$ & $735,897.42$ & $799,720.75$ \\
4 & 2,562 & $199,088.83$ & $176,460.40$ & $237,913.42$ & $185,750.43$ & $199,596.50$ \\
5 & 10,242 & $49,801.37$ & $44,123.63$ & $59,942.43$ & $46,638.43$ & $49,880.62$ \\
6 & 40,962 & $12,452.17$ & $11,031.44$ & $15,015.28$ & $11,700.00$ & $12,500.00$ \\
7 & 163,842 & $3,113.16$ & $2,757.89$ & $3,755.66$ & $2,919.71$ & $3,114.73$ \\
8 & 655,362 & 778.30 & 689.48 & 939.35 & 729.62 & 778.67 \\
9 & $2,621,442$ & 194.57 & 172.37 & 234.84 & 182.34 & 194.60 \\
\hline
\end{tabular}


The grid area was normalized according to Equation (15) to compare the differences at various resolutions.

$$
\widetilde{A}=\frac{A \times n v}{12 \times R^{2}}
$$

The normalized minimum area $\widetilde{A}_{\text {min }}$ and maximum area $\widetilde{A}_{\max }$ of different spherical icosahedral grids are listed in Table 2, and their curves are illustrated in Figure 5. Ratios between the minimum and maximum area, $r_{A}=A_{\min } / A_{\max }$, of different spherical icosahedral grids at different resolutions are presented in Figure 6.

Table 2. The normalized area and the ratios between the $A_{\min }$ and $A_{\max }$.

\begin{tabular}{|c|c|c|c|c|c|c|c|c|}
\hline \multicolumn{2}{|c|}{ Level } & 3 & 4 & 5 & 6 & 7 & 8 & 9 \\
\hline \multicolumn{2}{|c|}{$\widetilde{A}_{\text {avg }}$} & 1.047 & 1.047 & 1.047 & 1.047 & 1.047 & 1.047 & 1.047 \\
\hline \multirow{3}{*}{ NOPT } & $\widetilde{A}_{\min }$ & 0.930 & 0.928 & 0.930 & 0.928 & 0.928 & 0.928 & 0.928 \\
\hline & $\widetilde{A}_{\max }$ & 1.218 & 1.251 & 1.260 & 1.263 & 1.263 & 1.264 & 1.264 \\
\hline & $r_{A}$ & 0.764 & 0.742 & 0.740 & 0.735 & 0.735 & 0.734 & 0.734 \\
\hline \multirow{3}{*}{ SPRG } & $\widetilde{A}_{\min }$ & 0.879 & 0.848 & 0.820 & 0.803 & 0.791 & 0.781 & 0.776 \\
\hline & $\widetilde{A}_{\max }$ & 1.080 & 1.079 & 1.080 & 1.081 & 1.083 & 1.087 & 1.092 \\
\hline & $r_{A}$ & 0.814 & 0.786 & 0.760 & 0.743 & 0.730 & 0.718 & 0.711 \\
\hline \multirow{3}{*}{$\mathrm{HR}$} & $\widetilde{A}_{\text {min }}$ & 0.998 & 1.008 & 1.012 & 1.014 & 1.013 & 1.016 & 1.016 \\
\hline & $\widetilde{A}_{\max }$ & 1.062 & 1.066 & 1.068 & 1.068 & 1.069 & 1.070 & 1.070 \\
\hline & $r_{A}$ & 0.940 & 0.946 & 0.950 & 0.949 & 0.948 & 0.950 & 0.950 \\
\hline \multirow{3}{*}{ SCVT } & $\widetilde{A}_{\min }$ & 0.861 & 0.812 & 0.760 & 0.719 & 0.677 & 0.639 & 0.599 \\
\hline & $\widetilde{A}_{\max }$ & 1.082 & 1.081 & 1.080 & 1.081 & 1.081 & 1.081 & 1.081 \\
\hline & $r_{A}$ & 0.796 & 0.751 & 0.710 & 0.665 & 0.626 & 0.591 & 0.554 \\
\hline \multirow{3}{*}{ XU } & $\widetilde{A}_{\min }$ & 0.861 & 0.812 & 0.760 & 0.719 & 0.676 & 0.638 & 0.600 \\
\hline & $\widetilde{A}_{\max }$ & 1.082 & 1.081 & 1.080 & 1.081 & 1.082 & 1.082 & 1.082 \\
\hline & $r_{A}$ & 0.796 & 0.751 & 0.710 & 0.665 & 0.625 & 0.590 & 0.555 \\
\hline \multirow{3}{*}{ OURS } & $\widetilde{A}_{\min }$ & 0.970 & 0.977 & 0.981 & 0.984 & 0.982 & 0.982 & 0.981 \\
\hline & $\widetilde{A}_{\max }$ & 1.054 & 1.050 & 1.049 & 1.051 & 1.048 & 1.048 & 1.047 \\
\hline & $r_{A}$ & 0.920 & 0.930 & 0.940 & 0.936 & 0.937 & 0.937 & 0.937 \\
\hline
\end{tabular}

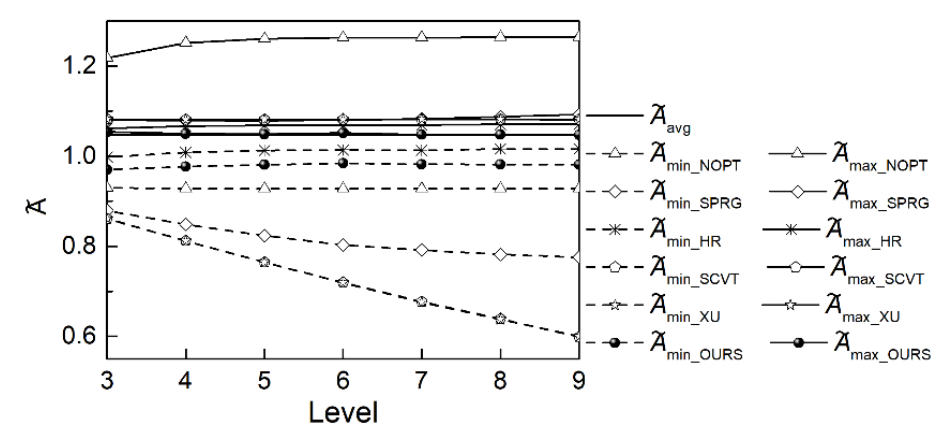

Figure 5. $\widetilde{A}_{\min }$ and $\widetilde{A}_{\max }$ of different grids.

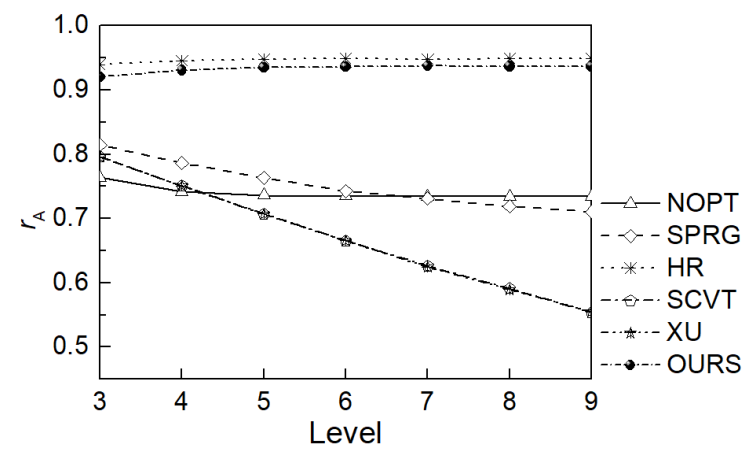

Figure 6. Ratios between $A_{\min }$ and $A_{\max }$ of different grids. 
The maximum grid area, minimum grid area and their ratios indicate the ranges of grid area. The closer the ratio is to one, the smaller the area range is, which indicates a more uniform grid area. The normalized minimum and maximum areas of all grids except the SCVT grid and XU grid gradually have converged as the resolution increases (Figures 5 and 6 and Tables 1 and 2). The area range of SCVT grid and XU grid are the same and the biggest among all grids at level 9. The one of NOPT grid is followed and that of HR grid is the smallest. The range of OURS grid is only expanded by $1.20 \%$ compared with HR grid. Same as the range, ratios of SCVT grid and XU grid are non-convergence as resolution increases and have decreased to 0.554 and 0.555 at level 9, respectively. The ratios of SPRG grid and NOPT grid have converged slowly to 0.711 and 0.734 , respectively. On the contrary, the ones of HR grid and OURS grid have increased as resolution increases and converged to 0.950 and 0.937 , respectively at the same resolution.

To further emphasize the spherical distribution characteristic of grid area uniformity, taking the grid at level 9 as an example, the number of grids in different intervals of grid area relative deviation calculated by Equation (15) are counted in Table 3.

Table 3. The number of grids in different $D_{\text {area }}$ interval of different grids.

\begin{tabular}{|c|c|c|c|c|c|c|}
\hline Interval (\%) & NOPT & SPRG & HR & SCVT & $\mathbf{X U}$ & OURS \\
\hline$[-100,-38.0]$ & 0 & 0 & 0 & 0 & 0 & 0 \\
\hline$(-38.0,-34.0)$ & 0 & 0 & 0 & 162 & 162 & 0 \\
\hline$(-34.0,-30.0)$ & 0 & 0 & 0 & 0 & 0 & 0 \\
\hline$(-30.0,-26.0)$ & 0 & 0 & 0 & 960 & 960 & 0 \\
\hline$(-26.0,-22.0)$ & 0 & 162 & 0 & 1,920 & 1,920 & 0 \\
\hline$(-22.0,-18.0)$ & 0 & 960 & 0 & 2,880 & 3,840 & 0 \\
\hline$(-18.0,-14.0)$ & 0 & 4,800 & 0 & 14,400 & 13,440 & 0 \\
\hline$(-14.0,-10.0)$ & 192 & 34,560 & 0 & 42,240 & 42,240 & 0 \\
\hline$(-10.0,-6.0)$ & 436,770 & 180,480 & 0 & 112,320 & 115,200 & 12 \\
\hline$(-6.0,-2.0)$ & $1,132,800$ & 630,720 & 22,786 & 314,880 & 317,760 & 0 \\
\hline$(-2.0,2.0)$ & 366,240 & 887,520 & 2598,480 & $1,403,040$ & $1,341,584$ & $2,621,430$ \\
\hline$(2.0,6.0)$ & 60,480 & 647,040 & 176 & 728,640 & 784,336 & 0 \\
\hline$(6.0,10.0)$ & 0 & 235,200 & 0 & 0 & 0 & 0 \\
\hline$(10.0,14.0)$ & 380,160 & 0 & 0 & 0 & 0 & 0 \\
\hline$(14.0,18.0)$ & 96,000 & 0 & 0 & 0 & 0 & 0 \\
\hline$(18.0,22.0)$ & 148,800 & 0 & 0 & 0 & 0 & 0 \\
\hline$(22.0,100.0)$ & 0 & 0 & 0 & 0 & 0 & 0 \\
\hline total & $2,621,442$ & $2,621,442$ & $2,621,442$ & $2,621,442$ & $2,621,442$ & $2,621,442$ \\
\hline
\end{tabular}

Refine these intervals to further demonstrate the area features of OURS grid (Table 4). Where, $N$ is the number of grids in different intervals, and $p$ is grid proportions.

The cumulative grid proportions of $\left|D_{\text {area }}\right|$ are depicted in Table 5, and Figure 7 $\left(\left|D_{\text {area }}\right|<0.08 \%\right)$.

The $D_{\text {area }}$ of all grids are in $[-38.0 \%, 22.0 \%]$ (Table 3). The ones of HR grid and OURS grid are mainly in $(-2.0 \%, 2.0 \%)$, which is smaller than other four grids. There are more than $90 \%$ grids with $D_{\text {area }}$ in $(-0.06 \%, 0.06 \%)$ in OURS grid. The cumulative proportions have increased logarithmically as $\left|D_{\text {area }}\right|$ increases, in which the increasing rate of OURS grid is the fastest and that of NOPT grid is the slowest (Figure 7). The cumulative proportions of OURS grid with $\left|D_{\text {area }}\right|$ of less than $0.044 \%$ is more than $90.00 \%$, and the ones of other five grids are only $0.02 \%$ (NOPT grid), $0.62 \%$ (SPRG grid), $13.24 \%$ (HR grid), $0.37 \%$ (SCVT grid), and $1.25 \%$ (XU grid). Although the area ratio of HR grid is bigger than OURS grid, the proportion of grid with smaller is far less than that of OURS grid.

Meanwhile, the spherical distributions of the area relative deviations at level 9 are shown in Figure 8, where the lighter the color is, the closer the grid area deviation is to zero. 
Table 4. The number of grids in refined $D_{\text {area }}$ interval of OURS grid.

\begin{tabular}{ccc}
\hline Interval (\%) & $N$ & $p \mathbf{( \% )}$ \\
\hline$[-100,-0.18)$ & 72 & 0.00 \\
{$[-0.18,-0.14)$} & 60 & 0.00 \\
{$[-0.14,-0.10)$} & 17,695 & 0.68 \\
{$[-0.10,-0.06)$} & 44,733 & 1.71 \\
{$[-0.06,-0.02)$} & 495,834 & 18.91 \\
{$[-0.02,0.02)$} & $1,342,129$ & 51.20 \\
$(-0.02,0.60)$ & 720,919 & 27.50 \\
$(0.06,100]$ & 0 & 0 \\
total & $2,621,442$ & 100.00 \\
\hline
\end{tabular}

Table 5. The cumulative proportion of $\left|D_{\text {area }}\right|$ of different grids (\%).

\begin{tabular}{ccccccc}
\hline Interval (\%) & NOPT & SPRG & HR & SCVT & XU & OURS \\
\hline$[0,0.004)$ & 0 & 0 & 1.07 & 0 & 0.26 & 12.57 \\
{$[0.004,0.012)$} & 0.02 & 0.07 & 3.27 & 0.07 & 0.59 & 33.35 \\
{$[0.012,0.02)$} & 0.02 & 0.22 & 5.87 & 0.07 & 0.73 & 51.20 \\
{$[0.02,0.028)$} & 0.02 & 0.22 & 8.63 & 0.22 & 0.93 & 66.98 \\
{$[0.028,0.036)$} & 0.02 & 0.51 & 11.06 & 0.22 & 1.25 & 81.04 \\
{$[0.036,0.044)$} & 0.02 & 0.62 & 13.24 & 0.37 & 1.25 & 92.56 \\
{$[0.044,0.052)$} & 0.02 & 0.77 & 15.10 & 0.66 & 1.39 & 96.17 \\
{$[0.052,0.06)$} & 0.02 & 0.99 & 16.73 & 0.92 & 1.39 & 97.61 \\
{$[0.06,0.068)$} & 0.02 & 1.65 & 22.95 & 2.38 & 1.76 & 99.32 \\
{$[0.068,0.076)$} & 0.02 & 2.23 & 28.11 & 3.04 & 2.16 & 99.99 \\
{$[0.076,0.3)$} & 0.02 & 4.69 & 43.54 & 6.23 & 5.35 & 99.99 \\
{$[0.3,0.5)$} & 0.02 & 7.87 & 56.64 & 10.25 & 9.87 & 99.99 \\
{$[0.5,0.7)$} & 0.02 & 11.17 & 66.66 & 14.39 & 14.25 & 99.99 \\
{$[0.7,0.9)$} & 0.02 & 14.39 & 74.64 & 18.82 & 18.53 & 99.99 \\
{$[0.9,2)$} & 13.97 & 33.86 & 99.12 & 53.52 & 51.18 & 99.99 \\
{$[6,26)$} & 59.49 & 82.6 & 100 & 93.33 & 93.32 & 100 \\
{$[26,100]$} & 100 & 100 & 100 & 100 & 100 & 100 \\
\hline
\end{tabular}

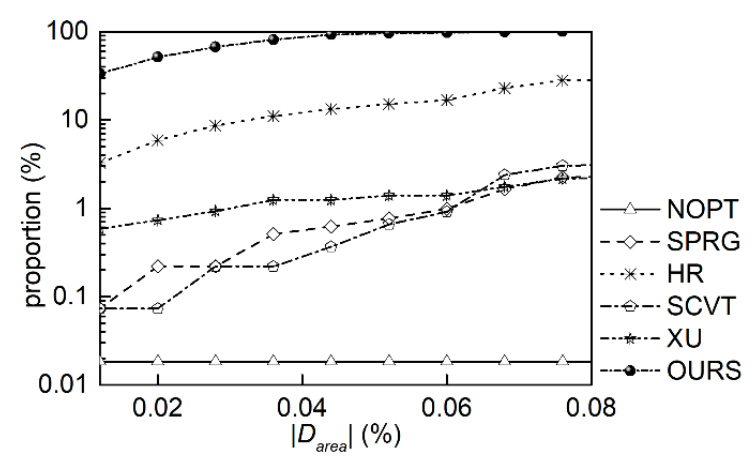

Figure 7. Curves of cumulative proportion for different grids at level $9\left(\left|D_{\text {area }}\right|<0.08 \%\right)$.

From Figure 8, the grids with maximum area deviation are the pentagons, and spherical distributions patterns of $D_{\text {area }}$ are symmetry. The distribution of NOPT grid has fractal characteristics and is not continuous. Because of the larger number of grids with larger $D_{\text {area, }}$ its distribution is shown by darker color. The distributions of the SPRG grid, SCVT grid and XU grid are similar. As the larger number of grids with smaller $D_{\text {area }}$ $(-0.06 \%, 0.06 \%)$ in OURS grid, its distribution is shown by the lightest color and some grids with larger $D_{\text {area }}$ are mainly located along the triangular boundaries. Grids with larger $D_{\text {area }}$ are also located around these boundaries, however, these regions are far larger than that of OURS grid. 


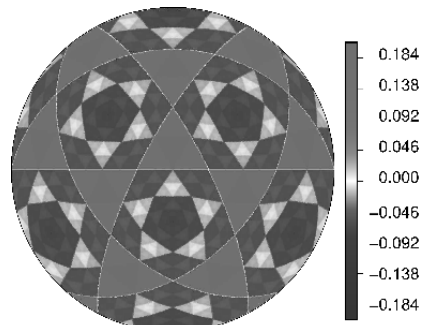

(a)

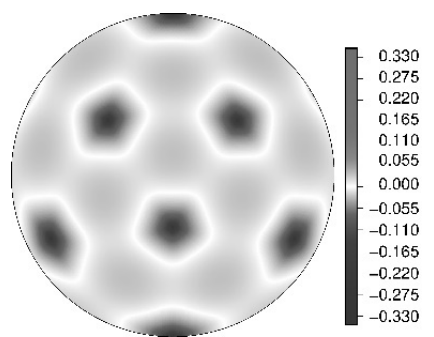

(d)

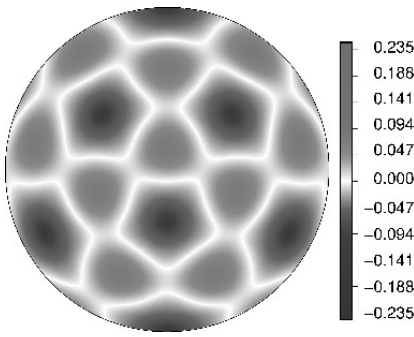

(b)

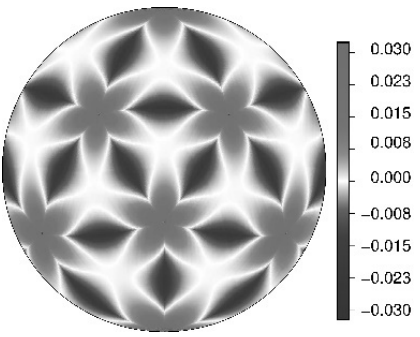

(c)

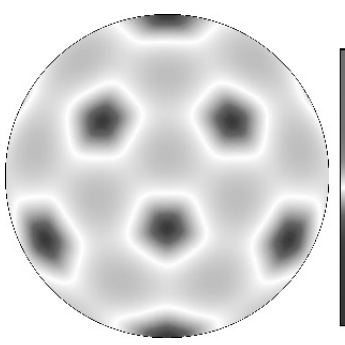

(e)

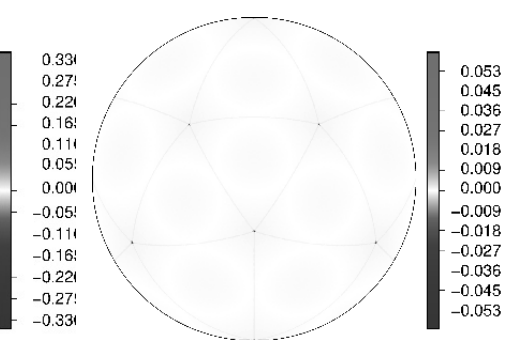

(f)

Figure 8. Spherical distributions of $D_{\text {area }}$ of different grids at level 9. (a) NOPT grid. (b) SPRG grid. (c) HR grid. (d) SCVT grid. (e) XU grid. (f) OURS grid.

\subsubsection{The Grid Interval Uniformity}

The geodesic distance, $d$, between grid points, the minimum distance, $d_{\min }$ and the maximum distance $d_{\max }$ (Table 6), the ratios between them (Table 7) and the grid length relative deviation (as Equation (16)) are calculated to describe the grid interval uniformity.

$$
D_{\text {length }}=\frac{L-L_{\mathrm{avg}}}{L_{\mathrm{avg}}}
$$

Table 6. $d_{\min }, d_{\max }, d_{\mathrm{avg}}$ of different grids (unit: $\mathrm{km}$ ).

\begin{tabular}{ccccccc}
\hline \multirow{2}{*}{ Level } & \multicolumn{3}{c}{ NOPT } & \multicolumn{3}{c}{ OURS } \\
\cline { 2 - 7 } & $\boldsymbol{d}_{\text {avg }}$ & $\boldsymbol{d}_{\text {min }}$ & $\boldsymbol{d}_{\text {max }}$ & $\boldsymbol{d}_{\text {avg }}$ & $\boldsymbol{d}_{\text {min }}$ & $\boldsymbol{d}_{\text {max }}$ \\
\hline 0 & 7529.85 & 7529.85 & 7529.85 & 7529.85 & 7529.85 & 7529.85 \\
\hline 1 & 3764.92 & 3526.83 & 4003.02 & 3764.92 & 3526.83 & 4003.02 \\
\hline 2 & 1914.33 & 1763.41 & 2079.28 & 1915.30 & 1739.23 & 2087.62 \\
\hline 3 & 961.22 & 881.71 & 1050.16 & 961.85 & 866.34 & 1059.12 \\
\hline 4 & 481.12 & 440.85 & 526.42 & 481.47 & 432.15 & 532.56 \\
\hline 5 & 240.62 & 220.43 & 263.38 & 240.81 & 215.84 & 267.18 \\
\hline 6 & 120.32 & 110.21 & 131.71 & 120.42 & 107.83 & 134.40 \\
\hline 7 & 60.16 & 55.11 & 65.86 & 60.20 & 54.13 & 67.12 \\
\hline 8 & 30.08 & 27.55 & 32.93 & 30.10 & 27.06 & 33.56 \\
\hline 9 & 15.04 & 13.78 & 16.47 & 15.05 & 13.53 & 16.78 \\
\hline
\end{tabular}


Table 7. Normalized distance and ratios between the $d_{\min }$ and $d_{\max }$ of different grids.

\begin{tabular}{|c|c|c|c|c|c|c|c|c|}
\hline \multicolumn{2}{|c|}{ Level } & 3 & 4 & 5 & 6 & 7 & 8 & 9 \\
\hline \multicolumn{2}{|c|}{$\widetilde{d}_{\mathrm{avg}}$} & 1.182 & 1.182 & 1.182 & 1.182 & 1.182 & 1.182 & 1.182 \\
\hline \multirow{3}{*}{ NOPT } & $\widetilde{d}_{\min }$ & 1.107 & 1.107 & 1.107 & 1.107 & 1.107 & 1.107 & 1.107 \\
\hline & $\widetilde{d}_{\max }$ & 1.319 & 1.322 & 1.323 & 1.323 & 1.323 & 1.323 & 1.323 \\
\hline & $r_{d}$ & 0.839 & 0.837 & 0.837 & 0.837 & 0.837 & 0.837 & 0.837 \\
\hline \multirow{3}{*}{ SPRG } & $\widetilde{d}_{\min }$ & 1.077 & 1.059 & 1.043 & 1.017 & 1.017 & 0.999 & 0.983 \\
\hline & $\tilde{d}_{\max }$ & 1.276 & 1.279 & 1.279 & 1.284 & 1.285 & 1.279 & 1.259 \\
\hline & $r_{d}$ & 0.844 & 0.828 & 0.815 & 0.802 & 0.791 & 0.781 & 0.781 \\
\hline \multirow{3}{*}{ HR } & $\widetilde{d}_{\min }$ & 1.076 & 1.074 & 1.074 & 1.074 & 1.074 & 1.074 & 1.075 \\
\hline & $\widetilde{d}_{\max }$ & 1.351 & 1.361 & 1.364 & 1.364 & 1.365 & 1.364 & 1.382 \\
\hline & $r_{d}$ & 0.796 & 0.789 & 0.787 & 0.787 & 0.787 & 0.787 & 0.778 \\
\hline \multirow{3}{*}{ SCVT } & $\widetilde{d}_{\min }$ & 1.065 & 1.035 & 1.005 & 0.975 & 0.946 & 0.917 & 0.890 \\
\hline & $\tilde{d}_{\max }$ & 1.275 & 1.277 & 1.277 & 1.277 & 1.277 & 1.277 & 1.280 \\
\hline & $r_{d}$ & 0.835 & 0.810 & 0.787 & 0.763 & 0.741 & 0.718 & 0.695 \\
\hline \multirow{3}{*}{$X U$} & $\widetilde{d}_{\min }$ & 1.065 & 1.035 & 1.005 & 0.975 & 0.945 & 0.916 & 0.888 \\
\hline & $\widetilde{d}_{\max }$ & 1.275 & 1.277 & 1.277 & 1.277 & 1.277 & 1.277 & 1.276 \\
\hline & $r_{d}$ & 0.835 & 0.810 & 0.787 & 0.763 & 0.740 & 0.717 & 0.696 \\
\hline \multirow{3}{*}{ OURS } & $\widetilde{d}_{\min }$ & 1.088 & 1.085 & 1.084 & 1.083 & 1.087 & 1.091 & 1.091 \\
\hline & $\widetilde{d}_{\max }$ & 1.330 & 1.337 & 1.342 & 1.350 & 1.349 & 1.354 & 1.353 \\
\hline & $r_{d}$ & 0.818 & 0.812 & 0.808 & 0.802 & 0.806 & 0.806 & 0.806 \\
\hline
\end{tabular}

The grid distance was normalized according to Equation (17) to compare the differences at various resolutions (Table 7 and Figure 9).

$$
\widetilde{d}=\frac{d \times 2^{\text {Level }}}{R}
$$

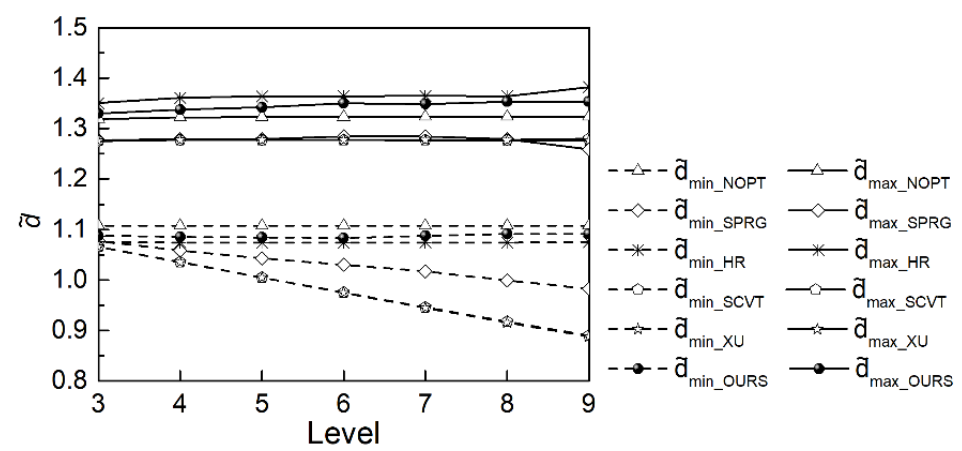

Figure 9. The normalized minimum and maximum distance of different grids.

Ratios between the minimum and maximum distances, $r_{d}=d_{\min } / d_{\max }$, of different grids at different resolutions are calculated in Table 7 and presented in Figure 10.

Same as the normalized area, $\widetilde{d}_{\min }, \widetilde{d}_{\max }$ and ratios indicate the ranges of grid interval. The closer the ratio is to one, the smaller the interval range is, which indicates a more uniform grid interval. The $\widetilde{d}_{\min }, \widetilde{d}_{\max }$ of the all grids except the SCVT grid and XU grid have gradually converged as the resolution increases (Figures 9 and 10 and Tables 6 and 7). The ratios of SCVT grid and XU grid are non-convergence as resolution increases, and ones of SPRG grid and HR grid have converged to 0.781 and 0.778 at level 9, respectively, the ratio of OURS grid is larger than them.

The number of grids in different intervals of grid length relative deviation have been counted (Table 8) to emphasize the spherical distribution characteristic of grid interval uniformity. 


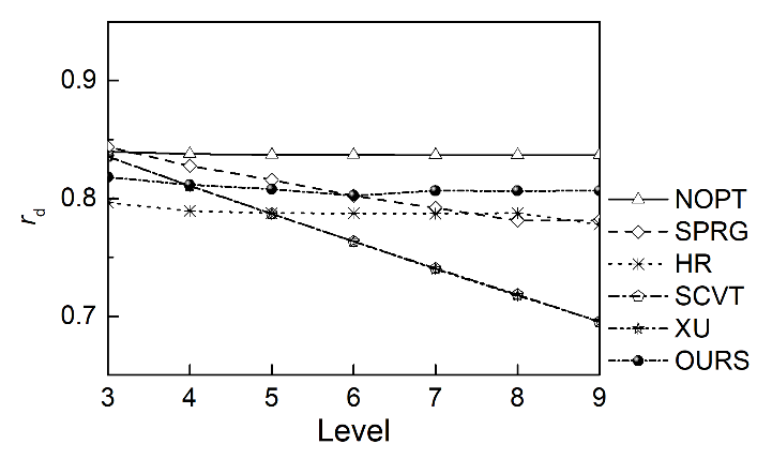

Figure 10. Ratios between minimum and maximum distance of different grids.

Table 8. The number of grids with different $D_{\text {length }}$ interval of different grids.

\begin{tabular}{ccccccc}
\hline & NOPT & SPRG & HR & SCVT & XU & OURS \\
\hline$[-100,-15]$ & 0 & 0 & 0 & 0 & 0 & 0 \\
$(-15,-13]$ & 0 & 0 & 0 & 60 & 60 & 0 \\
$(-13,-11]$ & 0 & 0 & 0 & 120 & 120 & 0 \\
$(-11,-9]$ & 0 & 0 & 0 & 360 & 420 & 0 \\
$(-9,-7]$ & 0 & 5,730 & 0 & 720 & 1,020 & 0 \\
$(-7,-5]$ & 0 & 28,800 & 0 & 2,640 & 2,280 & 0 \\
$(-5,-3]$ & 0 & 151,680 & 0 & 100,800 & 106,030 & 0 \\
$(-3,-1]$ & $1,488,960$ & 620,160 & 0 & 301,632 & 301,632 & 0 \\
$(-1,1)$ & 446,880 & 966,240 & $2,447,056$ & $1,536,160$ & $1,471,680$ & $2,621,430$ \\
{$[1,3)$} & 60,480 & 679,680 & 174,374 & 678,950 & 738,200 & 0 \\
{$[3,5)$} & 0 & 168,960 & 0 & 0 & 0 & 0 \\
{$[5,7)$} & 446,400 & 192 & 0 & 0 & 0 & 0 \\
{$[7,9)$} & 144,960 & 0 & 0 & 0 & 0 & 0 \\
{$[9,11)$} & 33,750 & 0 & 0 & 0 & 0 & 0 \\
{$[11,13)$} & 0 & 0 & 0 & 0 & 0 & 0 \\
{$[13,15)$} & 0 & 0 & 0 & 0 & 0 & 0 \\
{$[15,17)$} & 12 & 0 & 0 & 0 & 0 & 0 \\
{$[17,19)$} & 0 & 0 & 0 & 0 & 0 & 12 \\
{$[19,21)$} & 0 & 0 & 12 & 0 & 0 & 0 \\
{$[21,100]$} & 0 & 0 & 0 & 0 & 0 & 0 \\
total & $2,621,442$ & $2,621,442$ & $2,621,442$ & $2,621,442$ & $2,621,442$ & $2,621,442$ \\
\hline & & & & & &
\end{tabular}

There are the similar characteristics of HR and OURS grid from Table 8, so the intervals is refined to further reveal their differences in Table 9. Where, $N$ is the number of grids in different intervals, and $p$ is grid proportions.

Table 9. The number of grids with refined $D_{\text {length }}$ interval of HR and OURS grid.

\begin{tabular}{ccccc}
\hline \multirow{2}{*}{ Interval (\%) } & \multicolumn{2}{c}{ HR } & \multicolumn{2}{c}{ OURS } \\
\cline { 2 - 5 } & $\boldsymbol{N}$ & $\boldsymbol{p} \mathbf{( \% )}$ & 0 & $\boldsymbol{p} \mathbf{( \% )}$ \\
\hline$[-100,-1.1]$ & 16 & 0.00 & 0 & 0 \\
$(-1.1,-0.9]$ & 46,592 & 1.78 & 0 & 0 \\
$(-0.9,-0.7]$ & 137,776 & 5.26 & 0 & 0 \\
$(-0.7,-0.5]$ & 835,680 & 31.88 & 325,328 & 12.41 \\
$(-0.5,-0.3]$ & 468,576 & 17.87 & 659,536 & 25.16 \\
$(-0.3,-0.1]$ & 288,704 & 11.01 & 676,512 & 25.81 \\
$(-0.1,0.1)$ & 211,232 & 8.06 & 705,728 & 26.92 \\
{$[0.1,0.3)$} & 166,432 & 6.35 & 234,528 & 8.95 \\
{$[0.3,0.5)$} & 134,192 & 5.12 & 19,798 & 0.76 \\
{$[0.5,0.7)$} & 110,118 & 4.20 & 0 & 0 \\
{$[0.7,0.9)$} & 91,328 & 3.48 & 0 & 0 \\
{$[0.9,1.0)$} & 73,328 & 2.80 & 0 & 0 \\
{$[1.1,1.3)$} & 50,640 & 1.93 & & \\
\hline
\end{tabular}


Table 9. Cont.

\begin{tabular}{ccccc}
\hline \multirow{2}{*}{ Interval (\%) } & \multicolumn{2}{c}{ HR } & \multicolumn{3}{c}{ OURS } \\
\cline { 2 - 5 } & $N$ & $p(\%)$ & 0 & $p(\%)$ \\
\hline$[1.3,1.5)$ & 6,816 & 0.26 & 0 & 0 \\
{$[1.5,1.7)$} & 0 & 0 & 12 & 0 \\
{$[1.7,2.3)$} & 0 & 0 & 0 & 0 \\
{$[2.3,100]$} & 12 & 0 & $2,621,442$ & 100 \\
total & $2,621,442$ & 100 & \\
\hline
\end{tabular}

The cumulative proportions of grid with $\left|D_{\text {length }}\right|$ are depicted in Table 10 and Figure 11. Because of higher cumulative proportion in OURS grid in a smaller interval, only a part of results with $\left|D_{\text {length }}\right|<1.00 \%$ are demonstrated.

Table 10. The cumulative proportion of $\left|D_{\text {length }}\right|$ of different grids (\%).

\begin{tabular}{ccccccc}
\hline $\begin{array}{c}\text { Interval } \\
(\%)\end{array}$ & NOPT & SPRG & HR & SCVT & XU & OURS \\
\hline$[0,0.02)$ & 0 & 0.51 & 2.20 & 0.73 & 1.32 & 5.02 \\
{$[0.02,0.10)$} & 0.02 & 3.41 & 11.01 & 4.06 & 4.32 & 25.81 \\
{$[0.10,0.18)$} & 0.79 & 5.93 & 20.37 & 7.54 & 7.47 & 45.63 \\
{$[0.18,0.26)$} & 1.78 & 9.01 & 30.87 & 10.77 & 10.33 & 66.58 \\
{$[0.26,0.34)$} & 3.86 & 11.65 & 44.40 & 13.84 & 13.40 & 86.02 \\
{$[0.34,0.42)$} & 3.86 & 14.10 & 65.49 & 17.80 & 16.96 & 97.28 \\
{$[0.42,0.50)$} & 3.86 & 17.36 & 75.17 & 21.64 & 20.47 & 99.24 \\
{$[0.50,0.58)$} & 5.51 & 20.43 & 80.03 & 25.20 & 24.45 & 99.96 \\
{$[0.58,0.66)$} & 5.58 & 23.80 & 83.90 & 29.66 & 28.66 & 99.99 \\
{$[0.66,0.74)$} & 7.82 & 26.97 & 87.00 & 34.42 & 32.75 & 99.99 \\
{$[0.74,0.82)$} & 12.54 & 30.34 & 89.58 & 40.17 & 37.39 & 99.99 \\
{$[0.82,2.00)$} & 22.72 & 66.59 & 99.99 & 90.00 & 89.23 & 99.99 \\
{$[2.00,5.00)$} & 76.15 & 98.67 & 99.99 & 97.62 & 97.62 & 99.99 \\
{$[5.00,7.00)$} & 92.30 & 99.63 & 99.99 & 98.97 & 98.83 & 99.99 \\
{$[7.00,100]$} & 100 & 100 & 100 & 100 & 100 & 100 \\
\hline
\end{tabular}

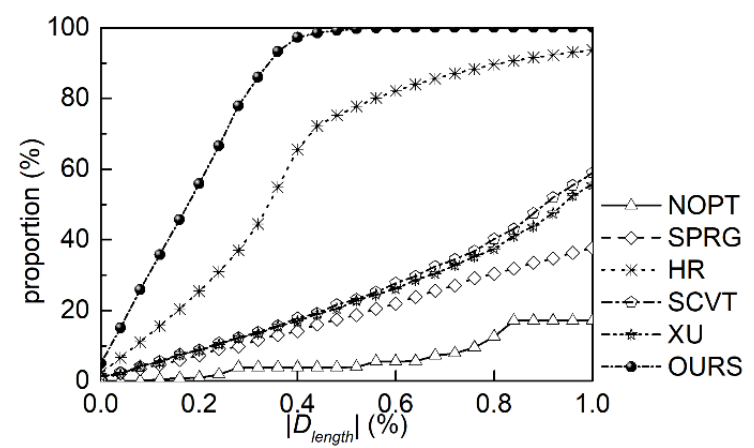

Figure 11. Curves of cumulative proportion with $\left|D_{\text {length }}\right|<1.00 \%$ for different grids at level 9 .

The $D_{\text {length }}$ of all grids are in $[-15 \%, 21 \%]$ (Table 8$)$, and the ones of HR grid and OURS grid are mainly in $(-1 \%, 1 \%)$. The proportion of grid in this range of OURS grid is about 7 times that of NOPT grid, although the interval ratio of NOPT grid is bigger than that of OURS grid. The cumulative proportions also increase logarithmically as $\left|D_{\text {length }}\right|$ increases, in which the increasing rate in OURS grid is the fastest and that of NOPT grid is the slowest. The cumulative proportions of OURS grid is more than $99.00 \%$ with $\left|D_{\text {length }}\right|$ less than $0.50 \%$. Ones of other grids are only $3.86 \%$ (NOPT grid) $17.36 \%$ (SPRG grid), $75.17 \%$ (HR grid), $21.64 \%$ (SCVT grid), and $20.47 \%$ (XU grid) in the same range.

Meanwhile, the spherical distributions of $D_{\text {length }}$ are shown in Figure 12 at level 9. 


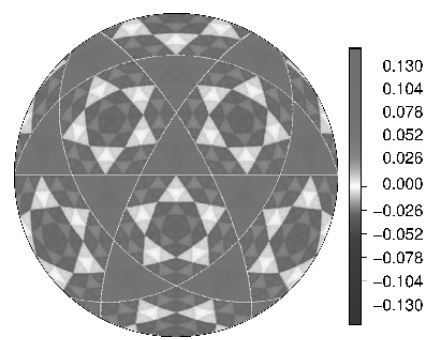

(a)

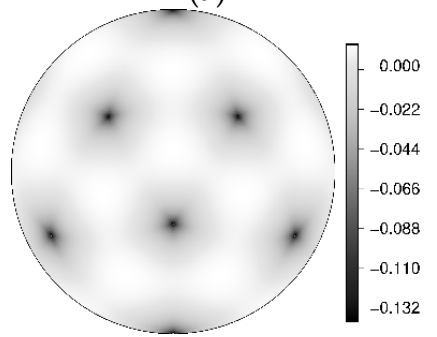

(d)

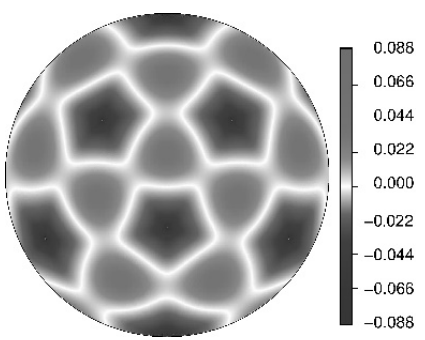

(b)

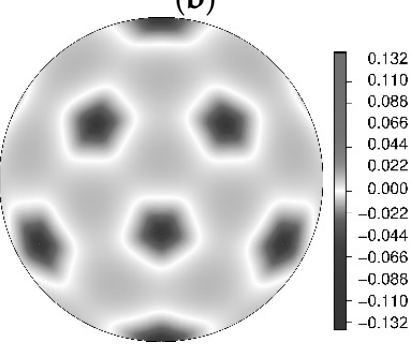

(e)

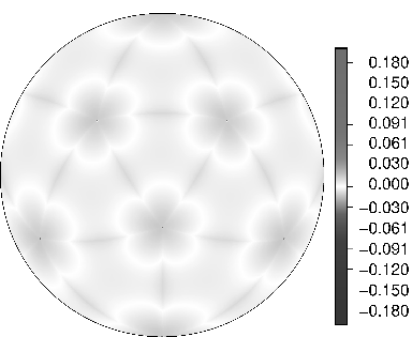

(c)

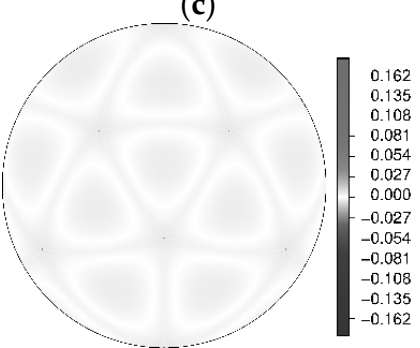

(f)

Figure 12. Spherical distributions of $D_{\text {length }}$ at level 9. (a) NOPT grid. (b) SPRG grid. (c) HR grid. (d) SCVT grid. (e) XU grid. (f) OURS grid.

Same as spherical distribution of grid area, the grids with maximum $D_{\text {length }}$ are these pentagons, and distributions of all grids are symmetry. Near these pentagons, the lengths of grids are less than the average length and shown by darker color in all grids. The distribution of NOPT grid has fractal characteristics and is not continuous, too. The $D_{\text {length }}$ in the remaining grids are reduced from the pentagons to triangular centers. The grids with larger $D_{\text {length }}$ are mainly located along the triangular boundaries in OURS grid, and those grids are spread like pentagon or star from these pentagons.

\subsection{The Numerical Accuracy Evaluation}

Spherical Laplacian operators of scalar field (as Equation (18)) is discretized to evaluate numerical accuracy on different grids.

$$
f(\lambda, \varphi)=\cos \lambda \times \cos ^{4} \varphi
$$

where $\lambda$ and $\varphi$ are the longitude and latitude of point on the sphere, respectively.

The $L_{2}$-norm error (as Equation (19)), $L_{\infty}$-norm error (as Equation (20)) of discretization have been calculated (Tables 11 and 12 and Figures 13 and 14).

$$
\begin{gathered}
L_{2}=\sqrt{\left(\frac{1}{\sum_{i=1}^{n v} A_{i}}\left(\sum_{i=1}^{n v} A_{i}\left(f_{i}^{\text {num }}-f_{i}^{\text {ana }}\right)^{2}\right)\right)} \\
L_{\infty}=\max _{i=1, n v}\left|f_{i}^{\text {num }}-f_{i}^{\text {ana }}\right|
\end{gathered}
$$

where $f^{\text {ana }}$ and $f^{\text {num }}$ are the analytical and numerical solutions of Laplacian operator, respectively.

Table 11. $L_{2}$-norm error of Laplacian operator of different grids.

\begin{tabular}{cccccccc}
\hline & $\mathbf{2}$ & $\mathbf{3}$ & $\mathbf{4}$ & $\mathbf{5}$ & $\mathbf{6}$ & $\mathbf{7}$ & $\mathbf{8}$ \\
\hline NOPT & $1.31 \times 10^{-1}$ & $3.78 \times 10^{-2}$ & $1.20 \times 10^{-2}$ & $4.49 \times 10^{-3}$ & $1.96 \times 10^{-3}$ & $9.26 \times 10^{-4}$ & $4.52 \times 10^{-4}$ \\
SPRG & $1.33 \times 10^{-1}$ & $3.56 \times 10^{-2}$ & $9.47 \times 10^{-3}$ & $2.74 \times 10^{-3}$ & $9.30 \times 10^{-4}$ & $3.84 \times 10^{-4}$ & $1.47 \times 10^{-4}$ \\
HR & $1.41 \times 10^{-1}$ & $3.79 \times 10^{-2}$ & $9.85 \times 10^{-3}$ & $2.58 \times 10^{-3}$ & $7.23 \times 10^{-4}$ & $2.05 \times 10^{-4}$ & $6.05 \times 10^{-5}$ \\
SCVT & $1.33 \times 10^{-1}$ & $3.55 \times 10^{-2}$ & $9.47 \times 10^{-3}$ & $2.81 \times 10^{-3}$ & $1.04 \times 10^{-3}$ & $4.61 \times 10^{-4}$ & $2.23 \times 10^{-4}$ \\
XU & $1.33 \times 10^{-1}$ & $3.55 \times 10^{-2}$ & $9.47 \times 10^{-3}$ & $2.81 \times 10^{-3}$ & $1.04 \times 10^{-3}$ & $4.61 \times 10^{-4}$ & $2.23 \times 10^{-4}$ \\
OURS & $1.38 \times 10^{-1}$ & $3.75 \times 10^{-2}$ & $9.78 \times 10^{-3}$ & $2.57 \times 10^{-3}$ & $6.98 \times 10^{-4}$ & $1.99 \times 10^{-4}$ & $5.42 \times 10^{-5}$ \\
\hline
\end{tabular}


Table 12. $L_{\infty}$-norm error of Laplacian operator of different grids.

\begin{tabular}{cccccccc}
\hline & $\mathbf{2}$ & $\mathbf{3}$ & $\mathbf{4}$ & $\mathbf{5}$ & $\mathbf{6}$ & $\mathbf{7}$ & $\mathbf{8}$ \\
\hline NOPT & $3.52 \times 10^{-1}$ & $1.28 \times 10^{-1}$ & $8.08 \times 10^{-2}$ & $8.89 \times 10^{-2}$ & $9.10 \times 10^{-2}$ & $9.10 \times 10^{-2}$ & $9.15 \times 10^{-2}$ \\
SPRG & $2.81 \times 10^{-1}$ & $7.62 \times 10^{-2}$ & $3.56 \times 10^{-2}$ & $3.23 \times 10^{-2}$ & $3.00 \times 10^{-2}$ & $2.70 \times 10^{-2}$ & $2.57 \times 10^{-2}$ \\
HR & $2.84 \times 10^{-1}$ & $8.67 \times 10^{-2}$ & $3.23 \times 10^{-2}$ & $1.41 \times 10^{-2}$ & $6.75 \times 10^{-3}$ & $3.31 \times 10^{-3}$ & $1.66 \times 10^{-3}$ \\
SCVT & $2.81 \times 10^{-1}$ & $7.61 \times 10^{-2}$ & $3.92 \times 10^{-2}$ & $3.88 \times 10^{-2}$ & $3.87 \times 10^{-2}$ & $3.87 \times 10^{-2}$ & $3.87 \times 10^{-2}$ \\
XU & $2.81 \times 10^{-1}$ & $7.61 \times 10^{-2}$ & $3.92 \times 10^{-2}$ & $3.88 \times 10^{-2}$ & $3.87 \times 10^{-2}$ & $3.87 \times 10^{-2}$ & $3.87 \times 10^{-2}$ \\
OURS & $2.85 \times 10^{-1}$ & $7.81 \times 10^{-2}$ & $2.93 \times 10^{-2}$ & $1.35 \times 10^{-2}$ & $6.59 \times 10^{-3}$ & $3.27 \times 10^{-3}$ & $1.59 \times 10^{-3}$ \\
\hline
\end{tabular}

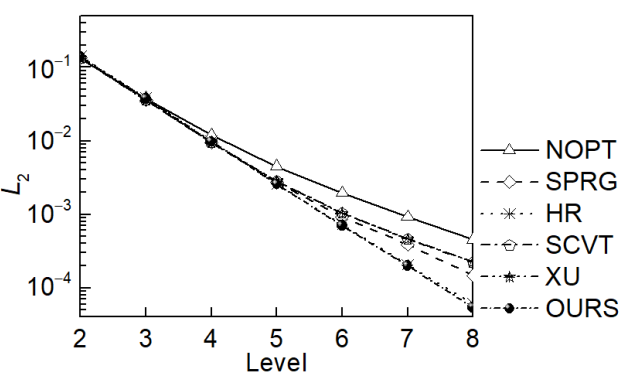

(a)

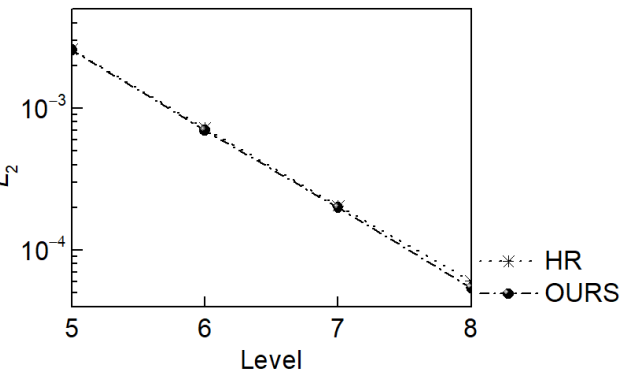

(b)

Figure 13. $L_{2}$-norm error of Laplacian operator. (a) $L_{2}$-norm error of all grids (b) $L_{2}$-norm error of OURS and HR grid from level 5 to 8.

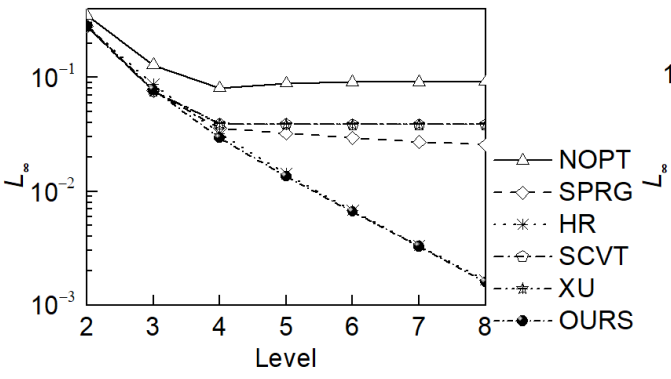

(a)

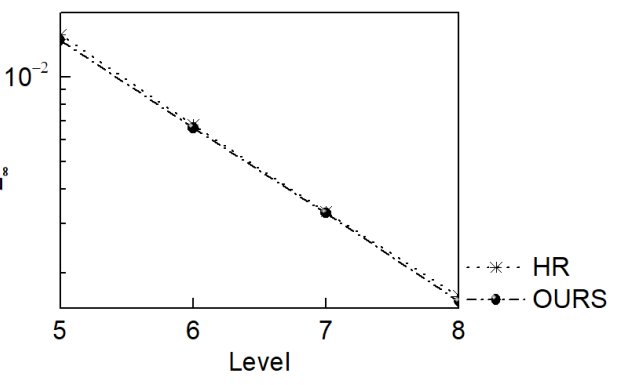

(b)

Figure 14. $L_{\infty}$-norm error of Laplacian operator. (a) $L_{\infty}$-norm error of all grids (b) $L_{\infty}$-norm error of OURS and HR grid from level 5 to 8.

The $\mathrm{L}_{2}$-norm errors and $\mathrm{L}_{\infty}$-norm errors of all grids have been reduced as resolution increase, ones of OURS grid and HR grid are smaller than the others. The $\mathrm{L}_{\infty}$-norm error (meaning the maximal error) of OURS grid has been reduced from $9.15 \times 10^{-2}$ (NOPT grid), $2.57 \times 10^{-2}$ (SPRG grid), $1.66 \times 10^{-2}$ (HR grid), $3.87 \times 10^{-2}$ (SCVT and XU grid) to $1.59 \times 10^{-2}$ at level 8 . The $\mathrm{L}_{2}$-norm error (meaning the RMS error) of OURS grid has been reduced from $4.52 \times 10^{-4}$ (NOPT grid), $2.03 \times 10^{-4}$ (SPRG grid), $6.05 \times 10^{-5}$ (HR grid), $2.33 \times 10^{-4}$ (SCVT and XU grid) to $5.86 \times 10^{-4}$ at the same grid resolution, in which the enhancement of average accuracy is nearly 8 times that of NOPT grid and has more than $11.62 \%$ compared to the HR grid.

Meanwhile, the spherical distributions of the Laplacian operator error on different grids are shown in Figure 15 at level 5.

Grids in NOPT grid with larger errors are mainly located the boundaries of refined icosahedral triangular cells, and that of all optimization grids are mainly located on the icosahedral triangular boundaries. In OURS grid, there are some fluctuations near icosahedral vertices and the middle part of boundaries, grids' error in these regions are smaller than that of HR grid. In addition, the error range of OURS grid is the smallest among all grids, and it has been narrowed to $84.81 \%$ of NOPT grid and $12.00 \%$ of HR grid. 


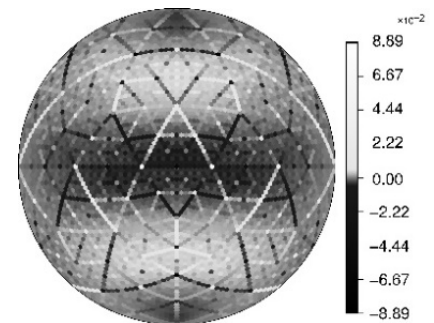

(a)

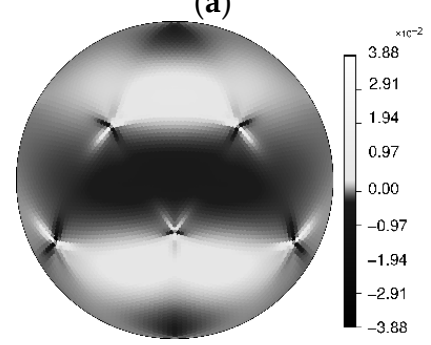

(d)

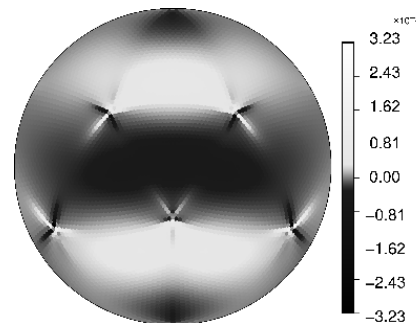

(b)

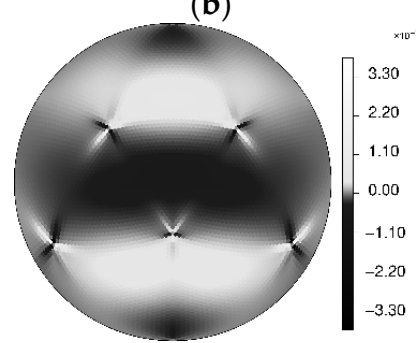

(e)

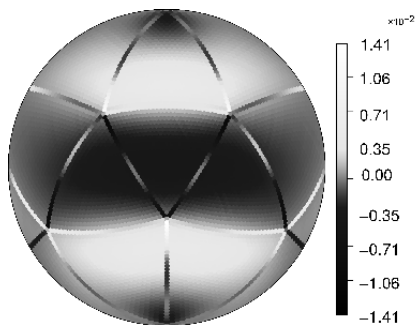

(c)

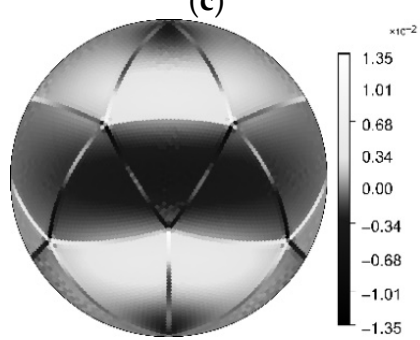

(f)

Figure 15. Spherical distributions of Laplacian operator error of different grids. (a) NOPT grid. (b) SPRG grid. (c) HR grid. (d) SCVT grid. (e) XU grid. (f) OURS grid.

\subsection{Discussion}

The raw grid (non-optimized grid) has been optimized by the proposed method. The area quasi-uniformity can be achieved by minimizing the grid area deviation cost, which improve the smoothness of grid area deviation to some extent. Although the grid area range of OURS grid is comparable to those of Heikes and Randall grid, the deformations of grid area and intervals of OURS grid are smoother, which is conducive to error control in simulation. In addition, the maximum error and RMS error of discritization of Laplacian operator have been decreased and converged as the resolution increases.

The grid quality is one of the aspects affecting the simulation accuracy. There is no high gradient of grid area and interval deformation in the optimized grid by our method, which can be helpful to improve the accuracy of discritization of Laplacian operator. A more extensive analysis of Laplace operator in a diffusion problem and some numerical experiments in terms of the accuracy and the numerical efficiency will be carried out in the future.

\section{Conclusions}

In this study, an overall uniformity and smoothness optimization method of the spherical icosahedral grid has been proposed based on the optimal transportation theory. The effectiveness of the proposed method was evaluated for grid uniformity and smoothness and the following conclusions may be drawn: (1) the area uniformity measured by the ratio between minimum and maximum grid area has been improved by $22.6 \%$ (SPRG grid), $38.3 \%$ (SCVT grid) and 38.2\% (XU grid), and can be comparable to the HR grid. The interval uniformity has also been increased by 2.5\% (SPRG grid), 2.8\% (HR grid), $11.1 \%$ (SCVT grid) and $11.0 \%$ (XU grid). (2) the smoothness of grid area deformation measured by the number of grids with grid area deviation of less than $0.05 \%$ has been enhanced by $79.32 \%$ (HR grid) and more than $90 \%$ compared to the SPRG grid, SCVT grid and XU grid. The smoothness of grid length deformation has also been increased by $24.07 \%$ (HR grid) and more than $75 \%$ compared to other three grids. Although the uniformity of grid optimized by the proposed method is comparable to the HR grid, the smoothness of grid deformation is improved. In addition, the average accuracy of Laplacian operator is slightly better than the HR grid.

In this study, we only evaluated the geometric uniformity of the optimized grid, and the truncation error of Laplacian operator. Future work will focus on the accuracy of 
Laplacian operator of different grids in a diffusion equation problem and some numerical experiments in terms of the accuracy and the numerical efficiency.

Author Contributions: F.L. formed the research idea, designed and conducted the experiment, drafted the manuscript; X.Z. co-designed the research, analyzed the results and revised the manuscript; W.S. revised the manuscript; Y.L. and Y.D. contributed to the grammar modification. All authors have read and agreed to the published version of the manuscript.

Funding: This research was funded by the National Key Research and Development Program of China (2018YFB0505301) and the National Natural Science Foundation of China (No. 41671394, No. 41671383).

Institutional Review Board Statement: Not applicable.

Informed Consent Statement: Not applicable.

Data Availability Statement: Data set available on request to corresponding authors.

Acknowledgments: The authors would like to sincerely thank the two anonymous reviewers whose insightful comments have helped to substantially improve the manuscript. We would like to acknowledge and thank Peixoto for providing the Heikes and Randall grid and SCVT grid.

Conflicts of Interest: The authors declare no conflict of interest.

\section{References}

1. Gassmann, A. A global hexagonal C-grid non-hydrostatic dynamical core (ICON-IAP) designed for energetic consistency. Q. J. R. Meteorol. Soc. 2013, 139, 152-175. [CrossRef]

2. Schubert, J.J.; Stevens, B.; Crueger, T. Madden-Julian oscillation as simulated by the MPI Earth System Model: Over the last and into the next millennium. J. Adv. Model. Earth Syst. 2013, 5, 71-84. [CrossRef]

3. Zängl, G.; Reinert, D.; Rípodas, P.; Baldauf, M. The ICON (ICOsahedral Non-hydrostatic) modelling framework of DWD and MPI-M: Description of the non-hydrostatic dynamical core. Q. J. R. Meteorol. Soc. 2015, 141, 563-579. [CrossRef]

4. Skamarock, W.C.; Klemp, J.B.; Duda, M.G.; Fowler, L.D.; Park, S.-H.; Ringler, T.D. A Multiscale Nonhydrostatic Atmospheric Model Using Centroidal Voronoi Tesselations and C-Grid Staggering. Mon. Weather. Rev. 2012, 140, 3090-3105. [CrossRef]

5. Satoh, M. Atmospheric Circulation Dynamics and General Circulation Models; Springer: Berlin/Heidelberg, Germany, 2014.

6. Dubos, T.; Dubey, S.; Tort, M.; Mittal, R.; Meurdesoif, Y.; Hourdin, F. DYNAMICO-1.0, an icosahedral hydrostatic dynamical core designed for consistency and versatility. Geosci. Model Dev. 2015, 8, 3131-3150. [CrossRef]

7. Walko, R.L.; Avissar, R. The Ocean-Land-Atmosphere Model (OLAM). Part I: Shallow-Water Tests. Mon. Weather Rev. 2008, 136, 4033-4044. [CrossRef]

8. Walko, R.L.; Avissar, R. The Ocean-Land-Atmosphere Model (OLAM). Part II: Formulation and Tests of the Nonhydrostatic Dynamic Core. Mon. Weather Rev. 2008, 136, 4045-4062. [CrossRef]

9. Revokatova, A.; Nikitin, M.; Rivin, G.; Rozinkina, I.; Nikitin, A.; Tatarinovich, E. High-Resolution Simulation of Polar Lows over Norwegian and Barents Seas Using the COSMO-CLM and ICON Models for the 2019-2020 Cold Season. Atmosphere 2021, $12,137$. [CrossRef]

10. Hsu, L.-H.; Chen, D.-R.; Chiang, C.-C.; Chu, J.-L.; Yu, Y.-C.; Wu, C.-C. Simulations of the East Asian Winter Monsoon on Subseasonal to Seasonal Time Scales Using the Model for Prediction Across Scales. Atmosphere 2021, 12, 865. [CrossRef]

11. Hay, H.C.F.C.; Matsuyama, I. Nonlinear tidal dissipation in the subsurface oceans of Enceladus and other icy satellites. Icarus 2018, 319, 68-85. [CrossRef]

12. Suzuki, K.; Nakajima, T.; Satoh, M.; Tomita, H.; Takemura, T.; Nakajima, T.; Stephens, G.L. Global cloud-system-resolving simulation of aerosol effect on warm clouds. Geophys. Res. Lett. 2008, 35, 610-616. [CrossRef]

13. Cheng, Y.; Dai, T.; Zhang, H.; Xin, J.; Chen, S.; Shi, G.; Nakajima, T. Comparison and evaluation of the simulated annual aerosol characteristics over China with two global aerosol models. Sci. Total Environ. 2021, 763, 143003-143017. [CrossRef] [PubMed]

14. Goto, D.; Kikuchi, M.; Suzuki, K.; Hayasaki, M.; Yoshida, M.; Nagao, T.M.; Choi, M.; Kim, J.; Sugimoto, N.; Shimizu, A.; et al. Aerosol model evaluation using two geostationary satellites over East Asia in May 2016. Atmos. Res. 2019, 217, 93-113. [CrossRef]

15. Yamashita, Y.; Takigawa, M.; Goto, D.; Yashiro, H.; Satoh, M.; Kanaya, Y.; Taketani, F.; Miyakawa, T. Effect of Model Resolution on Black Carbon Transport from Siberia to the Arctic Associated with the Well-Developed Low-Pressure Systems in September. J. Meteorol. Soc. Jpn. 2021, 99, 287-308. [CrossRef]

16. Cheng, Y.; Dai, T.; Goto, D.; Schutgens, N.A.J.; Shi, G.; Nakajima, T. Investigating the assimilation of CALIPSO global aerosol vertical observations using a four-dimensional ensemble Kalman filter. Atmos. Chem. Phys. 2019, 19, 13445-13467. [CrossRef]

17. Korn, P. Formulation of an unstructured grid model for global ocean dynamics. J. Comput. Phys. 2017, 339, 525-552. [CrossRef]

18. Peixoto, P.S. Accuracy analysis of mimetic finite volume operators on geodesic grids and a consistent alternative. J. Comput. Phys. 2016, 310, 127-160. [CrossRef] 
19. Peixoto, P.S.; Barros, S.R.M. Analysis of grid imprinting on geodesic spherical icosahedral grids. J. Comput. Phys. 2013, 237, 61-78. [CrossRef]

20. Wang, N.; Lee, J.L. Geometric Properties of the Icosahedral-Hexagonal Grid on the Two-Sphere. Soc. Ind. Appl. Math. 2011, 33, 2536-2559. [CrossRef]

21. Weller, H.; Thuburn, J.; Cotter, C.J. Computational modes and grid imprinting on five quasi-uniform spherical c-grids. Mon. Weather Rev. 2012, 140, 2734-2755. [CrossRef]

22. Cheong, H.; Kang, H. Eigensolutions of the spherical Laplacian for the cubed-sphere and icosahedral-hexagonal grids. Q. J. R. Meteorol. Soc. 2015, 141, 3383-3398. [CrossRef]

23. Miura, H.; Kimoto, M. A comparison of grid quality of optimized spherical hexagonal-pentagonal geodesic grids. Mon. Weather Rev. 2005, 133, 2817-2833. [CrossRef]

24. Subich, C.J. Higher-order finite volume differential operators with selective upwinding on the icosahedral spherical grid. J. Comput. Phys. 2018, 368, 21-46. [CrossRef]

25. Xu, G. Discrete Laplace-Beltrami Operator on Sphere and Optimal Spherical Triangulations. Int. J. Comput. Geom. Appl. 2006, 16, 75-93. [CrossRef]

26. Korn, P.; Linardakis, L. A conservative discretization of the shallow-water equations on triangular grids. J. Comput. Phys. 2018, 375, 871-900. [CrossRef]

27. Tomita, H.; Tsugawa, M.; Satoh, M.; Goto, K. Shallow Water Model on a Modified Icosahedral Geodesic Grid by Using Spring Dynamics. J. Comput. Phys. 2001, 174, 579-613. [CrossRef]

28. Tomita, H.; Satoh, M.; Goto, K. An Optimization of the Icosahedral Grid Modified by Spring Dynamics. J. Comput. Phys. 2002, 183, 307-331. [CrossRef]

29. Iga, S.; Tomita, H. Improved smoothness and homogeneity of icosahedral grids using the spring dynamics method. J. Comput. Phys. 2014, 258, 208-226. [CrossRef]

30. Iga, S. An equatorially enhanced grid with smooth resolution distribution generated by a spring dynamics method. J. Comput. Phys. 2017, 330, 794-809. [CrossRef]

31. Heikes, R.; Randall, D.A. Numerical Integration of the Shallow-Water Equations on a Twisted Icosahedral Grid. Part I: Basic Design and Results of Tests. Mon. Weather Rev. 1995, 123, 1862-1880. [CrossRef]

32. Heikes, R.; Randall, D.A. Numerical Integration of the Shallow-Water Equations on a Twisted Icosahedral Grid. Part II. A Detailed Description of the Grid and an Analysis of Numerical Accuracy. Mon. Weather Rev. 1995, 123, 1881-1887. [CrossRef]

33. Heikes, R.P.; Randall, D.A.; Konor, C.S. Optimized icosahedral grids: Performance of finite-difference operators and multigrid solver. Mon. Weather Rev. 2013, 141, 4450-4469. [CrossRef]

34. Du, Q.; Gunzburger, M.D.; Ju, L. Constrained centroidal Voronoi tessellations for surfaces. Siam J. Sci. Comput. 2003, 24, 1488-1506. [CrossRef]

35. Du, Q.; Gunzburger, M.D.; Ju, L. Voronoi-based finite volume methods, optimal Voronoi meshes, and PDEs on the sphere. Comput. Methods Appl. Mech. Eng. 2003, 192, 3933-3957. [CrossRef]

36. Ju, L.; Ringler, T.; Gunzburger, M. Voronoi Tessellations and Their Application to Climate and Global Modeling; Springer: Berlin/Heidelberg, Germany, 2011; Volume 80, pp. 313-342.

37. Du, Q.; Vance, F.; Max, G. Centroidal Voronoi Tessellations: Applications and Algorithms. SIAM Rev. 1999, 41, 637-676. [CrossRef]

38. Miura, H. Application of the Synchronized B Grid Staggering for Solution of the Shallow-Water Equations on the Spherical Icosahedral Grid. Mon. Weather Rev. 2019, 147, 2485-2509. [CrossRef]

39. Wang, N.; Bao, J.W.; Lee, J.L.; Moeng, F.; Matsumoto, C. Wavelet Compression Technique for High-Resolution Global Model Data on an Icosahedral Grid. J. Atmos. Ocean. Technol. 2015, 32, 1650-1667. [CrossRef]

40. Jubair, M.; Alim, U.; Röber, N.; Clyne, J.; Mahdavi-Amiri, A. Icosahedral Maps for a Multiresolution Representation of Earth Data. In Proceedings of the VMV'16 Proceedings of the Conference on Vision, Modeling and Visualization, Bayreuth, Germany, 10-12 October 2016.

41. Gu, X.; Luo, F.; Sun, J. Variational principles for Minkowski type problems, discrete optimal transport, and discrete Monge-Ampere equations. Math. Methods Solid State Superfluid Theory 2013, 20, 383-398. [CrossRef]

42. Cui, L.; Qi, X.; Wen, C.; Lei, N.; Li, X.; Zhang, M.; Gu, X. Spherical optimal transportation. Comput. -Aided Des. 2019, 115, 181-193. [CrossRef]

43. Tu, Y.; Wen, C.; Wen, Z.; Wu, J.F. Isometry Invariant Shape Descriptors for Abnormality Detection on Brain Surfaces Affected by Alzheimer's Disease. In Proceedings of the 2018 40th Annual International Conference of the IEEE Engineering in Medicine and Biology Society (EMBC), Honolulu, HI, USA, 17-21 July 2018.

44. Giri, A.; Choi, G.; Kumar, L. Open and closed anatomical surface description via hemispherical area-preserving map. Signal Process. 2020, 180, 107867-107880. [CrossRef]

45. Su, Z.; Zeng, W.; Wang, Y.; Lu, L.Z.; Gu, X.F. Shape Classification Using Wasserstein Distance for Brain Morphometry Analysis. In Information Processing in Medical Imaging (IPMI); Springer: Berlin/Heidelberg, Germany, 2015.

46. Su, Z.; Wang, Y.; Shi, R. Optimal mass transport for shape matching and comparison. IEEE Trans. Pattern Anal. Mach. Intell. 2015, 37, 2246-2259. [CrossRef] [PubMed]

47. Lei, N.; Su, K.; Cui, L.; Yau, S.-T.; Gu, X.D. A Geometric View of Optimal Transportation and Generative Model. Comput. Aided Geom. Des. 2019, 68, 1-21. [CrossRef] 